\title{
Cough symptom severity in patients with refractory or unexplained chronic cough: a systematic survey and conceptual framework
}

\author{
Elena Kum ${ }^{1}$, Gordon H. Guyatt ${ }^{1,2}$, Tahira Devji ${ }^{1}$, Yuting Wang ${ }^{1}$, Layla Bakaa ${ }^{3}$, Lucy Lan $\mathbb{C}^{4}$, Eva Liu ${ }^{4}$, \\ Alexander Mastrolonardo ${ }^{4}$, Rachel Couban $\mathbb{1}^{1}$, Paul M. O'Byrne $\mathbb{1}^{2,5}$ and Imran Satia $\mathbb{1}^{1,2,5}$
}

${ }^{1}$ Dept of Health Research Methods, Evidence, and Impact, McMaster University, Hamilton, ON, Canada. ${ }^{2}$ Dept of Medicine, McMaster University, Hamilton, ON, Canada. ${ }^{3}$ Dept of Psychology, Neuroscience, and Behaviour, McMaster University, Hamilton, ON, Canada. ${ }^{4}$ Michael G. DeGroote School of Medicine, McMaster University, Hamilton, ON, Canada. ${ }^{5}$ Firestone Institute for Respiratory Health, St Joseph's Healthcare, Hamilton, ON, Canada.

Corresponding author: Imran Satia (satiai@mcmaster.ca)

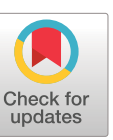

This version is distributed under the terms of the Creative Commons Attribution NonCommercial Licence 4.0. For commercial reproduction rights and permissions contact permissions@ersnet.org

This article has supplementary material available from err.ersjournals.com

Received: 19 April 2021 Accepted: 18 May 2021

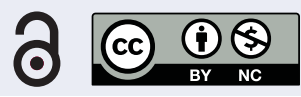

Shareable abstract (@ERSpublications)

The urge-to-cough (frequency and intensity) and the cough symptom (control, frequency, bout duration, intensity and sequelae) represent domains to assess cough severity in patients with refractory or unexplained chronic cough https://bit.ly/3feqBXZ

Cite this article as: Kum E, Guyatt GH, Devji T, et al. Cough symptom severity in patients with refractory or unexplained chronic cough: a systematic survey and conceptual framework. Eur Respir Rev 2021; 30: 210104 [DOI: 10.1183/16000617.0104-2021].

\section{Abstract}

Background Cough severity represents an important subjective endpoint in assessing the effectiveness of therapies for patients with chronic cough. Although cough-specific quality of life questionnaires exist, a widely available cough severity instrument with established measurement properties remains unavailable.

Aims To identify and summarise the results of studies reporting on the experience of patients with chronic cough and, in the process, develop a conceptual framework to inform development of a patient-reported outcome measurement (PROM) addressing cough severity.

Results We identified 61 eligible studies reporting on patient experience with chronic cough. Studies provided 82 potential items, of which 43 proved unique and relevant to cough severity. The urge-to-cough sensation and the cough symptom itself represented broad domains of cough severity. Two subdomains under urge-to-cough included frequency (1 item) and intensity (1 item). Five subdomains under cough symptoms included control (2 items), frequency (6 items), bout duration (1 item), intensity (8 items), and associated features/sequelae (24 items).

Conclusions Our systematic survey and conceptual framework identified items and domains of cough severity in patients with refractory or unexplained chronic cough. The results support item generation and content validity for a PROM assessing cough severity.

\section{Introduction}

Chronic cough lasting for more than 8 weeks is a common health problem that affects between $2-18 \%$ of adults worldwide [1]. Although clinicians can often identify and effectively treat known causes of cough (including asthma, COPD, bronchiectasis, gastro-oesophageal reflux disease and upper airway cough syndromes), up to $60 \%$ of patients experience persistent coughing despite treatment targeting these underlying conditions (refractory chronic cough), while in others, no cause can be identified (unexplained chronic cough) [2-4].

Chronic cough can result in important impairment in quality of life. Physical symptoms associated with chronic cough include chest pain, urinary incontinence, cough syncope and headaches [5]. Patients may also experience depression, anxiety, sleep disorders and social disability [5]. Improving quality of life in these patients requires investigation and administration of effective therapies. 
Objective measurement of cough frequency represents the current gold standard to assess the efficacy of novel therapies for refractory or unexplained chronic cough in clinical trial settings, particularly for regulatory approval. Limitations of cough frequency monitoring include, however, restricted timeframe (typically $>24 \mathrm{~h}$ ), burden for patients, and expense. Together, these render the technology unfeasible for use in routine clinical practice. Although objective cough frequency is an important endpoint and a dominant aspect of severity, there may be other patient-important factors that contribute to patients' overall perception of cough severity.

Patient-reported outcomes (PROs) represent measures of patients' health status that are obtained from direct patient inquiry without interpretation by a clinician or anyone else [6]. PROs including the Leicester Cough Questionnaire (LCQ) [7], Chronic Cough Impact Questionnaire (CCIQ) [8], Cough Specific Quality of Life Questionnaire (CQLQ) [9], Cough Assessment Test (COAT) [10], and Cough Evaluation Test [11] provide insights into patients’ subjective experience of chronic cough. Most of these measures focus on the impact of cough symptoms on quality of life and are thus limited in that they only indirectly address cough severity. This is important because improvement in the subjective experience of cough may be not only due to cough suppression, but also due to central neuromodulatory effects from agents such as opioids, pregabalin and gabapentin that diminish the aversive nature of the cough experience. Thus, alongside cough-specific quality of life, full insight into therapeutic efficacy requires assessment of cough symptom severity itself.

The visual analogue scale (VAS), in which patients use a $100 \mathrm{~mm}$ scale ranging from "no cough" to "the worst cough severity”, provides a measure of patients' experience of cough severity [12]. Limitations of the cough severity VAS include, however, lack of evidence supporting its measurement properties, difficulty comparing VAS scores between and within individuals due to lack of interpretability, and limitations of single-item instruments in fully capturing complex patient experiences [13].

The cough symptom score (CSS), a two-part diary that includes questions related to cough frequency, severity, and impact during the day and night, provides another subjective measure of cough severity [14]. Clinical experience with this instrument is, however, limited, and the tool lacks a conceptual framework. Validity, responsiveness, and interpretability of the CSS also remain untested.

In contrast to the cough severity VAS and the CSS, the Cough Severity Diary (CSD), a seven-item daily diary including domains of frequency, intensity and disruption, has undergone psychometric testing [15-17]. It is, however, currently a proprietary questionnaire that's use for clinical or research purposes remain restricted. Furthermore, it lacks conceptual clarity in that the domain "disruption” measures cough impact on daytime activities and sleep, which represent measurements of cough-specific quality of life rather than cough symptom severity. A widely available instrument with established measurement properties that subjectively measures cough symptom severity rather than cough-specific quality of life remains unavailable.

Apart from the CSD, an important limitation of the cough severity measures we have discussed is that they were not informed by a thorough systematic survey of the available literature documenting patients' experience of chronic cough. Failure to conduct a comprehensive survey risks omission of important items and limits the development of a conceptual framework to optimally define the underlying construct and domains for a cough severity instrument [18-20]. Therefore, we conducted a systematic survey to identify both relevant domains and items to assess cough severity.

\section{Methods}

We conducted a systematic survey following a protocol registered on Open Science Framework (https://osf. io/tnjba). This report adheres to preferred reporting items for systematic reviews and meta-analyses (PRISMA) standards [21]. A steering group (E. Kum, G.H. Guyatt and I. Satia) developed the plan for the study, the detailed methodology, and led the development of the conceptual framework.

\section{Data sources and search}

We searched the databases of MEDLINE, EMBASE, CINAHL, PsycINFO, PubMed and HaPI from January 2007 to August 2020. The search strategy, adapted to each database and without language restrictions, included terms representing cough and terms representing PRO measurements (PROMs) (supplementary table 1). To identify relevant studies prior to 2007, we referred to a systematic survey [16] that included related articles within the literature from 2002 to 2006, as well as reviewed reference lists of all eligible studies from our updated search. 


\section{Eligibility criteria}

We included studies meeting any of the following criteria: 1) studies addressing item generation of a cough-specific PROM (including PROMs that assess cough symptom severity, urge-to-cough severity and/ or their impacts); 2) qualitative studies (interviews and focus groups) addressing the experience of chronic cough; 3) cross-sectional studies addressing the experience of chronic cough; and 4) longitudinal cohort studies (prospective or retrospective) addressing the prognosis of chronic cough, in which cough was the independent predictor of a patient-important outcome. Eligible studies reported at least one relevant domain or item that informed the concept of cough severity.

We included studies that enrolled adult patients (age $\geqslant 18$ years) with chronic cough of any aetiology. Chronic cough was defined as cough lasting more than 8 weeks [22]. Studies including mixed cough populations (i.e. chronic cough, subacute cough and acute cough) or that defined chronic cough with a threshold duration of less than 8 weeks were deemed eligible if the study reported data stratified for patients with cough lasting more than 8 weeks, or if $\geqslant 80 \%$ of the patients had cough lasting more than 8 weeks.

We excluded the following studies: 1) randomised clinical trials in which cough serves as a primary or secondary endpoint; 2) case reports related to chronic cough; 3) reviews addressing chronic cough; 4) editorials; 5) letters to the editor; 6) conference abstracts; 7) studies that failed to explicitly define chronic cough or failed to report the average duration of chronic cough; and 8) studies including only patients with lung cancer, cystic fibrosis, pulmonary hypertension or heart failure.

\section{Study selection}

Using Covidence systematic review software, a pair of reviewers, following training and calibration exercises, first independently screened all titles and abstracts, and subsequently the full texts of studies identified as potentially eligible. Reviewers resolved disagreements by discussion and, when necessary, by adjudication with a third reviewer (E. Kum, G.H. Guyatt or I. Satia).

\section{Data extraction}

For each eligible study, a pair of reviewers, following training and calibration exercises, extracted data independently using a standardised, pilot tested data extraction form (supplementary appendix). Reviewers collected information on study characteristics (study design and sample size) and patient characteristics (country, setting, type of care, age, sex, smoking habits, smoking pack-years, causes of chronic cough, and duration of cough). For studies addressing item generation or development of a PROM, we extracted information on the questionnaires' target population, content (construct(s) assessed, number of items, number of domains, and labelling of domains), and format (scoring and recall period).

Reviewers extracted statements informing candidate items and domains of cough severity. Informative statements included those on: 1) precipitants and sensations leading up to a cough; 2) characteristics of coughing while it is happening; and 3) symptoms in the immediate period (approximately 10 mins) after coughing that patients perceive to be caused by coughing. Reviewers extracted relevant statements verbatim from the articles.

Statements deemed uninformative of cough severity included those that referred to domains and items related to cough-specific quality of life, including cough impacts on daily life, mental/psychological health, and social life. Statements referring to symptoms that may co-occur with coughing but that are not due to coughing itself were also considered uninformative. Reviewers resolved discrepancies by discussion and, when necessary, by adjudication with a third reviewer. To ensure consistency of judgements, one researcher (E. Kum) was a member of all reviewer pairs. A senior investigator (G.H. Guyatt or I. Satia) adjudicated any conceptual issues that proved particularly challenging.

\section{Qualitative synthesis}

In parallel with the data extraction process, a steering group (E. Kum, G.H. Guyatt and I. Satia) developed a separate list of domains and items using our own phrasing (a taxonomy), adding domains and items as new themes from extracted statements emerged. For each item or domain, the taxonomy specified a collection of common terms and statements used to convey the same or related ideas. By assigning the most closely related keywords, reviewers used the taxonomy to organise the statements they extracted according to its meaning and content [23]. Reviewers could suggest new items or domains when a quotation did not fit existing ones. The continuous updating of the taxonomy provided a method to involve all reviewers in the qualitative synthesis process. 
After completion of data extraction, the steering group reviewed the taxonomy and suggested improvements to the wording. Potential items suggested by reviewers could be eliminated if judged ineligible by the steering group. These ineligible items included those that provided redundant, uninterpretable, or irrelevant concepts. The steering group subsequently developed a draft conceptual framework that, through interactive discussion, led to arrangement of eligible items and domains, and to some domains being subsumed within existing categories, given less prominence. We used vote counting, comprising the number of studies mentioning either the domain or an item under that domain, to identify the frequency with which authors mentioned items or domains presented in the final conceptual framework.

\section{Results}

After screening 6425 titles and abstracts and 334 full texts, 61 studies proved eligible (figure 1). Supplementary table 2 documents studies excluded at the full text level.

Table 1 presents the characteristics of the included studies that typically used cross-sectional designs $(n=31 ; 50.8 \%)$ and were conducted in the UK $(n=20 ; 32.8 \%)$, USA $(n=13 ; 21.3 \%)$, and Australia $(n=7$; $11.5 \%)$. Studies commonly defined chronic cough with a threshold duration of $>8$ weeks $(n=40 ; 65.6 \%)$.

Typical patients proved to be females aged $>50$ years who had never smoked and were receiving care from secondary or tertiary care settings (table 2). One qualitative study interviewed a sample of international respiratory specialists, rather than patients with chronic cough [36].

The search identified eligible studies addressing item generation or development of a number of cough-specific PROMs: CCIQ [8], Cough and Sputum Questionnaire [32], COAT [10], Cough Evaluation Test (CET) [11], CSD [15], Cough Severity Index [65], CQLQ [9], CSS [14], Hull Airway Reflux
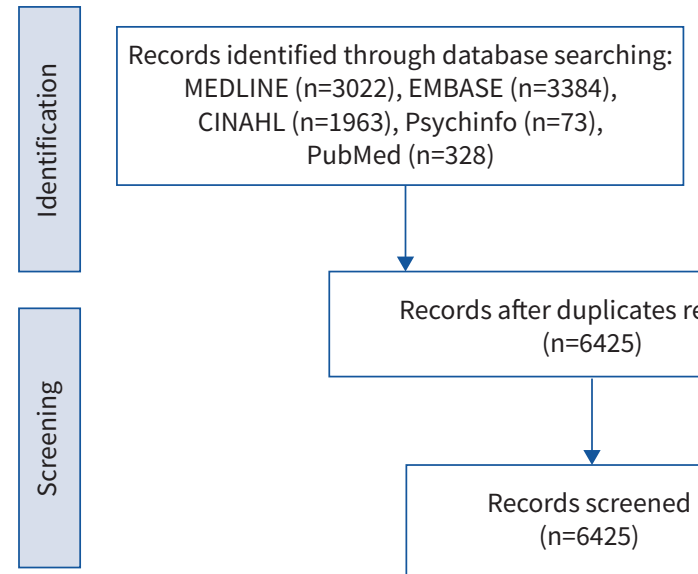

Records after duplicates removed $(n=6425)$
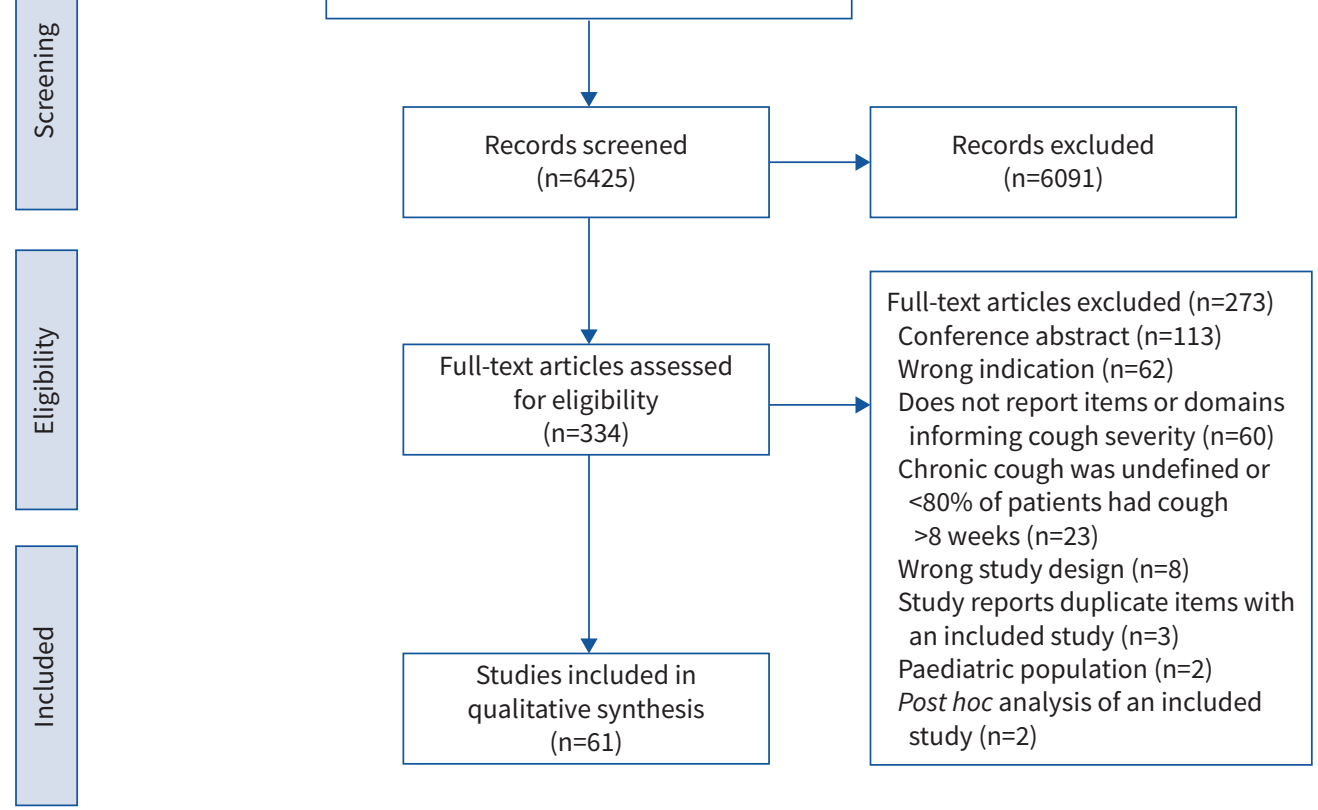

FIGURE 1 Study selection flow chart. 
TABLE 1 Characteristics of included studies

\begin{tabular}{|c|c|c|c|c|}
\hline First author [ref.] & $\begin{array}{l}\text { Total } \\
\text { sample } \\
\text { size }\end{array}$ & Country & Definition of chronic cough & Study type \\
\hline AdAms [24] & 3960 & Australia & Cough lasting $>12$ weeks & Prospective cohort study \\
\hline BAIARDINI [8] & 166 & Italy & Cough lasting $>3$ weeks & $\begin{array}{c}\text { Study addressing item generation of a } \\
\text { PROM (Chronic Cough Impact } \\
\text { Questionnaire) }\end{array}$ \\
\hline BIRRING [7] & 104 & UK & Cough lasting $>3$ weeks & $\begin{array}{l}\text { Study addressing item generation of a } \\
\text { PROM (Leicester Cough Questionnaire) }\end{array}$ \\
\hline CAMPI [25] & 1204 & Italy & Cough lasting $>8$ weeks & Cross-sectional study \\
\hline ChamberLain [26] & 1120 & $\begin{array}{l}\text { UK, Germany, France, Italy, Poland, } \\
\text { Russia, Ukraine, Latvia, Lithuania, }\end{array}$ & Cough lasting $>8$ weeks & Cross-sectional study \\
\hline
\end{tabular}

Moldova, Romania, Bulgaria, Turkey,

Greece, Czech Republic, Austria,

Switzerland, Spain, Portugal, Ireland,

Iceland, Norway, Sweden, Belgium,

Netherlands, Denmark, Cyprus,

\begin{tabular}{|c|c|c|c|}
\hline \multicolumn{4}{|c|}{ Luxembourg } \\
\hline CHAN [27] & 55 & Australia & Cough lasting $>8$ weeks \\
\hline Cheng [28] & 176 & Canada & NR \\
\hline ÇOLAK [29] & 14740 & Denmark & Cough lasting $>8$ weeks \\
\hline ÇOLAK [30] & 14669 & Denmark & Cough lasting $>8$ weeks \\
\hline CORNFORD [31] & 30 & USA & $\begin{array}{c}\text { Cough lasting }>3 \text { weeks and } \\
\text { who consulted due to coug } \\
\text { as the primary illness }\end{array}$ \\
\hline CRAWFORD [32] & 41 & Germany, Spain, France, Japan, USA & $\begin{array}{l}\text { Daily cough and sputum } \\
\text { production }>3 \text { months }\end{array}$ \\
\hline Decalmer [33] & 62 & UK & Cough lasting $>8$ weeks \\
\hline EVERETT [34] & 373 & UK & NR \\
\hline EVERETT [35] & 47 & UK & $\begin{array}{c}\text { Demonstrable abnormality } \\
\text { oesophageal manometry or } \\
\text { PH monitoring or both }\end{array}$ \\
\hline FARUQI [36] & 86 & UK, USA, Japan, Germany & NA \\
\hline FARUQI [37] & 25 & UK & Cough lasting $>6$ months \\
\hline FORD [38] & 3883 & UK & Cough lasting $>8$ weeks \\
\hline FRENCH [39] & 80 & USA & Cough lasting $>8$ weeks \\
\hline FRENCH [40] & 39 & USA & Cough lasting $>3$ weeks \\
\hline
\end{tabular}

\begin{tabular}{|c|c|c|c|c|}
\hline & & & & \\
\hline FUJIMURA [41] & 1000 & Japan & Cough lasting $>8$ weeks & Qualitative study \\
\hline HILTON [42] & 100 & UK & Cough lasting $>8$ weeks & Cross-sectional study \\
\hline Hsu [14] & 47 & UK & Cough lasting $>6$ months & $\begin{array}{l}\text { Study addressing item generation of a } \\
\text { PROM (Cough Symptom Score) }\end{array}$ \\
\hline HuLme [43] & 14 & UK & Cough lasting $>8$ weeks & Qualitative study \\
\hline KANG [44] & 447 & Korea & Cough lasting $>8$ weeks & Cross-sectional study \\
\hline KeLSALL [45] & 62 & UK & Cough lasting $>8$ weeks & Cross-sectional study \\
\hline KeLSALL [46] & 70 & UK & Cough lasting $>8$ weeks & Cross-sectional study \\
\hline Koo [10] & 401 & Korea & Cough lasting $>8$ weeks & $\begin{array}{l}\text { Study addressing item generation of a } \\
\text { PROM (Cough Assessment Test) }\end{array}$ \\
\hline KOSKELA [47] & 3697 & Finland & Cough lasting $>8$ weeks & Cross-sectional study \\
\hline KosKeLA [48] & 3695 & Finland & Cough lasting $>8$ weeks & Cross-sectional study \\
\hline KuZNAIR [49] & 136 & USA & Cough lasting $>3$ weeks & Cross-sectional study \\
\hline LAI [50] & 1162 & China & Cough lasting $>8$ weeks & Cross-sectional study \\
\hline LAN [51] & 164 & Australia & Cough lasting $>8$ weeks & Cross-sectional study \\
\hline LANDT [52] & 41545 & Denmark & Cough lasting $>8$ weeks & Prospective cohort study \\
\hline LÄTTI [53] & 3673 & Finland & Cough lasting $>8$ weeks & Cross-sectional study \\
\hline LeE $[54]$ & 49 & UK & Cough lasting $>8$ weeks & Cross-sectional study \\
\hline LeE [55] & 100 & UK & Cough lasting $>8$ weeks & Cross-sectional study \\
\hline LeE [56] & 32 & UK & Cough lasting $>8$ weeks & Cross-sectional study \\
\hline
\end{tabular}




\begin{tabular}{|c|c|c|c|c|}
\hline First author [ref.] & $\begin{array}{l}\text { Total } \\
\text { sample } \\
\text { size }\end{array}$ & Country & Definition of chronic cough & Study type \\
\hline MA [57] & 200 & China & Cough lasting $>8$ weeks & Prospective cohort study \\
\hline MeLLo [58] & 88 & USA & Cough lasting $>3$ weeks & Cross-sectional study \\
\hline MORICE [59] & 255 & UK & Cough lasting $>8$ weeks & $\begin{array}{c}\text { Study addressing item generation of a } \\
\text { PROM (Hull Airway Reflux } \\
\text { Questionnaire) }\end{array}$ \\
\hline MURRY [60] & 16 & USA & NR & Retrospective cohort study \\
\hline Osoo [61] & 112 & UK & Cough lasting $>8$ weeks & Prospective cohort study \\
\hline OzGe [62] & 165 & Turkey & Cough lasting $>4$ weeks & Cross-sectional study \\
\hline SATo [63] & 129 & Japan & NR & Cross-sectional study \\
\hline SHARIAT [64] & 360 & USA & NR & Cross-sectional study \\
\hline Shembel [65] & 365 & USA & Cough lasting $>8$ weeks & $\begin{array}{c}\text { Study addressing item generation of a } \\
\text { PROM (Cough Severity Index) }\end{array}$ \\
\hline SINHA [66] & 223 & UK & Cough lasting $>8$ weeks & Qualitative study \\
\hline SмITH [67] & 26 & UK & CSS daytime cough score $\geqslant 2$ & Cross-sectional study \\
\hline Song [68] & 857 & Korea & Cough lasting $>12$ weeks & $\begin{array}{l}\text { Cross-sectional analysis of a } \\
\text { prospective cohort study }\end{array}$ \\
\hline $\begin{array}{l}\text { TeRnesten-HASSEus } \\
{[69]}\end{array}$ & 121 & Sweden & Cough lasting $>8$ weeks & Cross-sectional study \\
\hline $\begin{array}{l}\text { VAN DE KERKHOVE } \\
{[70]}\end{array}$ & 51 & Belgium & Cough lasting $>8$ weeks & Prospective cohort study \\
\hline VAzQuez [71] & 1422 & USA & Cough lasting $>2$ years & Prospective cohort study \\
\hline VERNON [16] & 22 & USA & Cough lasting $>8$ weeks & Qualitative study \\
\hline VERNON [15] & 39 & USA & Cough lasting $>8$ weeks & $\begin{array}{c}\text { Study addressing item generation of a } \\
\text { PROM (Cough Severity Diary) }\end{array}$ \\
\hline VERTIGAN [72] & 38 & Australia & Cough lasting $>8$ weeks & $\begin{array}{c}\text { Study addressing item generation of a } \\
\text { PROM (Newcastle Laryngeal } \\
\text { Hypersensitivity Questionnaire) }\end{array}$ \\
\hline Vertigan [73] & 171 & Australia & Cough lasting $>8$ weeks & Cross-sectional study \\
\hline VERTIGAN [74] & 171 & Australia & Cough lasting $>8$ weeks & Cross-sectional study \\
\hline Vertigan [75] & 53 & Australia & Cough lasting $>8$ weeks & Cross-sectional study \\
\hline Won [76] & 478 & Korea & Cough lasting $>8$ weeks & Cross-sectional study \\
\hline $\mathrm{Xu}[77]$ & 87 & China & Cough lasting $>8$ weeks & Retrospective cohort study \\
\hline ZhAN [11] & 237 & China & Cough lasting $>8$ weeks & $\begin{array}{c}\text { Study addressing item generation of a } \\
\text { PROM (Cough Evaluation Test) }\end{array}$ \\
\hline
\end{tabular}

Questionnaire [59], LCQ [7], Newcastle Laryngeal Hypersensitivity Questionnaire [72], and Punum Ladder $[39,78]$. Supplementary table 3 lists the characteristics of these PROMs.

\section{Saturation of the taxonomy}

A taxonomy of potential items and domains was largely established after the first 20 studies. Few additional items or domains were added to the taxonomy when extracting the remaining 41 studies.

\section{Item identification}

From included studies, reviewers identified a total of 82 items. Each candidate item was carefully reviewed by the steering group for eligibility. Items judged ineligible on review included: those that represented objective physiologic measurements (i.e. compressive phase duration, oesophageal pressure, expiratory muscle activation, gastric pressure, and peak cough flow) rather than subjective measurements of cough intensity; conditions rather than symptoms associated with coughing (i.e. cardiac rhythm disturbance and subconjunctival haemorrhage); vague descriptions (i.e. worsening cough, character of cough); or concepts unrelated to cough severity (i.e. interference, disturbance, cough triggers, haemoptysis). Items referring to synonymous concepts (i.e. shortness of breath, dyspnoea, and breathlessness) were grouped into a single item. This resulted in 43 unique items reflected in our final taxonomy (table 3). 
TABLE 2 Characteristics of patients in included studies

\begin{tabular}{|c|c|c|c|c|c|c|c|c|c|c|c|}
\hline $\begin{array}{l}\text { First author } \\
\text { [ref.] }\end{array}$ & $\begin{array}{l}\text { Total } \\
\text { sample } \\
\text { size }\end{array}$ & $\begin{array}{l}\text { Sample } \\
\text { size of } \\
\text { patients } \\
\text { with } \\
\text { chronic } \\
\text { cough }\end{array}$ & $\begin{array}{l}\text { Type of } \\
\text { care/ } \\
\text { sample } \\
\text { type }\end{array}$ & $\begin{array}{c}\text { Age } \\
\text { (years) }\end{array}$ & $\begin{array}{l}\text { Female } \\
(\%)\end{array}$ & $\begin{array}{l}\text { Never } \\
\text { smoker } \\
(\%)\end{array}$ & $\begin{array}{l}\text { Current } \\
\text { smoker } \\
(\%)\end{array}$ & $\begin{array}{l}\text { Former } \\
\text { smoker } \\
(\%)\end{array}$ & $\begin{array}{l}\text { Smoking } \\
\text { pack history } \\
\text { (pack-years) }\end{array}$ & $\begin{array}{l}\text { Causes of } \\
\text { chronic cough } \\
\text { (\%) }\end{array}$ & $\begin{array}{l}\text { Duration of } \\
\text { chronic cough }\end{array}$ \\
\hline Adams [24] & 3960 & 812 & $\begin{array}{l}\text { Other; } \\
\text { general } \\
\text { population }\end{array}$ & NR & NR & NR & NR & NR & NR & $\begin{array}{l}13.5 \text { (asthma), } 7.3 \text { (CB), } \\
1.3 \text { (emphysema) }\end{array}$ & NR \\
\hline BAIARDINI [8] ${ }^{\#}$ & 166 & 166 & $\begin{array}{l}\text { Secondary/ } \\
\text { tertiary }\end{array}$ & 53.9 & 56.0 & 34.3 & 39.8 & 25.9 & NR & NR & $\begin{array}{l}25 \text { months } \\
\text { (sD: } 9 \text { months) }\end{array}$ \\
\hline BIRRING $[7]^{\natural}$ & 104 & 104 & $\begin{array}{l}\text { Secondary/ } \\
\text { tertiary }\end{array}$ & 57.0 & 62.5 & NR & NR & NR & NR & 100 (UCC) & $\begin{array}{l}65 \text { months (range: } \\
2.6-650 \text { months) }\end{array}$ \\
\hline CAMPI [25] & 1204 & 1204 & $\begin{array}{l}\text { Secondary/ } \\
\text { tertiary }\end{array}$ & 61.0 & 70.4 & 61.1 & 8.6 & 29.0 & NR & 1.74 (COPD) & 39 months \\
\hline ChamberLAin [26] & 1120 & 1120 & $\begin{array}{l}\text { Other; } \\
\text { general } \\
\text { population }\end{array}$ & 51.0 & 67.0 & NR & 16.5 & NR & NR & $\begin{array}{c}11.88 \text { (asthma), } 7.14 \text { (GORD), } \\
3.57 \text { (PND), } 8.75 \text { (bronchitis), } \\
\text { 4.38 (COPD), } 3.84 \text { (viral infection), } \\
2.41 \text { (cough hypersensitivity } \\
\text { syndrome), } 0.89 \text { (laryngitis), } \\
1.96 \text { (allergy), other }\end{array}$ & $\begin{array}{l}26-65 \text { months (range: } \\
2 \text { months to } 10+\text { years) }\end{array}$ \\
\hline CHAN [27] & 55 & 18 & NR & 53.0 & 32.7 & 100.0 & 0.0 & 0.0 & NR & $\begin{array}{l}100 \text { (sleep disordered breathing, no } \\
\text { known respiratory cause of cough) }\end{array}$ & $\begin{array}{l}67 \% \text { ( } \leqslant 8 \text { weeks) } \\
33 \% \text { (>8 weeks) }\end{array}$ \\
\hline Cheng [28] & 176 & NR & $\begin{array}{l}\text { Secondary/ } \\
\text { tertiary }\end{array}$ & 64.3 & 56.8 & NR & 2.8 & 59.7 & 8.17 & $\begin{array}{c}15.91 \text { (ACEi), } 76.70 \text { (GORD), } 11.93 \\
\text { (chronic sinus disease), } 28.98 \\
\text { (chronic heart disease), } 19.32 \\
\text { (non-ILD chronic lung disease) }\end{array}$ & 36.5 months \\
\hline ÇOLAK [29] & 14740 & 554 & $\begin{array}{l}\text { Other; } \\
\text { general } \\
\text { population }\end{array}$ & 58.2 & 54.5 & 45.6 & 10.4 & 43.1 & 14.87 & 12.64 (asthma), 87.36 (other) & NR \\
\hline ÇOLAK [30] & 14669 & 554 & $\begin{array}{l}\text { Other; } \\
\text { general } \\
\text { population }\end{array}$ & 58.2 & 55.1 & 46.0 & 10.5 & 43.5 & NR & $\begin{array}{c}2.53 \text { (asthma), } \\
0.18 \text { (bronchiectasis), } \\
2.89 \text { (GORD), } 1.26 \text { (UACS) }\end{array}$ & NR \\
\hline CORNFORD [31] & 30 & 30 & Primary & 48.0 & NR & NR & NR & NR & NR & NR & NR \\
\hline CRAWFORD $[32]^{+}$ & 41 & NR & NR & NR & NR & NR & NR & NR & NR & 100 (CB) & NR \\
\hline Decalmer [33] & 62 & 62 & $\begin{array}{l}\text { Secondary/ } \\
\text { tertiary }\end{array}$ & 54.9 & 62.9 & NR & 0.0 & 37.1 & NR & $\begin{array}{l}53.23 \text { (GORD), } 12.9 \text { (PND), } \\
9.68 \text { (asthma), } 3.23 \text { (EB), } \\
3.23 \text { (bronchiectasis), } \\
3.23 \text { (tracheopathia } \\
\text { osteochondroplastica) }\end{array}$ & $\begin{array}{l}71.5 \text { months (range: } \\
13-390 \text { months) }\end{array}$ \\
\hline EVERETt [34] & 373 & 373 & $\begin{array}{l}\text { Primary/ } \\
\text { secondary/ } \\
\text { tertiary }\end{array}$ & 65.3 & 73.0 & NR & 2.1 & 40.8 & 8 & $\begin{array}{l}24 \text { (asthma), } 66 \text { (no respiratory } \\
\text { diagnosis other than cough) }\end{array}$ & $\begin{array}{l}84.5 \text { months (range: } \\
0.63-949 \text { months) }\end{array}$ \\
\hline Everett [35] & 47 & 47 & $\begin{array}{l}\text { Secondary/ } \\
\text { tertiary }\end{array}$ & 54.3 & 55.3 & NR & NR & NR & NR & 100 (GORD) & NR \\
\hline
\end{tabular}


TABLE 2 Continued

First author

[ref.]

Total
sample

\section{Sample}

size of

patients

care/

$\begin{array}{cc}\text { Age } & \text { Female } \\ \text { (years) } & (\%)\end{array}$

\section{Never}

Current

Former
smoker

Smoking
pack history

smok
(\%)

(\%) (\%)

(\%)

(pack-years)

chronic

cough

type

Causes of

Duration of

chronic cough

chronic cough

(\%)

\begin{tabular}{|c|c|c|c|c|c|c|c|c|c|c|c|}
\hline FARUQI [36] & 86 & NA & NA & NA & NA & NA & NA & NA & NA & NA & NA \\
\hline FARUQI [37] & 25 & 25 & $\begin{array}{l}\text { Secondary/ } \\
\text { tertiary }\end{array}$ & 54.0 & 60.0 & 0.0 & $\mathrm{NR}$ & NR & NR & NR & 130 months \\
\hline FoRD [38] & 3883 & 481 & Primary & $40-49$ & 56.1 & NR & 21.7 & NR & NR & NR & NR \\
\hline FRENCH [39] & 80 & 80 & $\begin{array}{l}\text { Secondary/ } \\
\text { tertiary }\end{array}$ & 58.5 & 68.8 & 97.5 & $\mathrm{NR}$ & NR & NR & $\begin{array}{c}86.3 \text { (GORD), } 72.5 \text { (UACS), } 27.5 \\
\text { (asthma), } 5.0 \text { (drug induced), } \\
3.8 \text { (bronchiectasis), } \\
\text { 3.8 (postinfectious), } 2.5 \text { (NAEB), } \\
2.5 \text { (COPD), } 2.5 \text { (laryngeal sensory } \\
\text { neuropathy), } 1.3 \text { (CHF), } \\
1.3 \text { (bronchiolitis), } 1.3 \text { (infiltrate), } \\
1.3 \text { (suppurative airway infection), } \\
1.3 \text { (vocal cord dysfunction), } 1.3 \text { (ILD) }\end{array}$ & $\begin{array}{l}86 \text { months } \\
\text { (sD: } 123.7 \text { months) }\end{array}$ \\
\hline French [40] & 39 & 39 & $\begin{array}{l}\text { Secondary/ } \\
\text { tertiary }\end{array}$ & 54.0 & 82.1 & NR & NR & NR & NR & $\begin{array}{c}40 \text { (PND), } 36 \text { (GORD) } 15 \text { (asthma), } \\
3 \text { (bronchiectasis), } 3 \text { (pertussis), } \\
3 \text { (other) }\end{array}$ & $\begin{array}{c}56 \text { months } \\
\text { (sD: } 63 \text { months) }\end{array}$ \\
\hline FUJIMURA [41] & 1000 & 232 & $\begin{array}{l}\text { Other; } \\
\text { general } \\
\text { population }\end{array}$ & 55.3 & 38.2 & 41.6 & 22.2 & 36.1 & 566 & $\begin{array}{c}10.1 \text { (cold), } 18.5 \text { (asthma), } \\
7.6 \text { (CB), } 6.7 \text { (CVA), } 5.9 \text { (GORD), } \\
\text { sinusitis, } 6.7 \text { (pulmonary } \\
\text { emphysema), pneumonia, atopic } \\
\text { cough, lung cancer, COPD, } \\
17.6 \text { (CB), } 15.1 \text { (unknown) }\end{array}$ & 3.8 weeks \\
\hline HILTON [42] & 100 & 100 & $\begin{array}{l}\text { Secondary/ } \\
\text { tertiary }\end{array}$ & 60.0 & 71.0 & NR & 0.0 & NR & NR & NR & $\begin{array}{c}91 \text { (IQR: } \\
\text { 52-195 months) }\end{array}$ \\
\hline Hsu [14] & 47 & 14 & $\begin{array}{l}\text { Secondary/ } \\
\text { tertiary }\end{array}$ & 49.1 & 57.1 & NR & NR & NR & NR & $\begin{array}{l}14.29 \text { (GORD), } 21.43 \text { (PND due to } \\
\text { paranasal sinusitis or rhinosinusitis), } \\
4 \text { (responsive to inhaled steroid } \\
\text { therapy, no wheeze or shortness of } \\
\text { breath), } 5 \text { (UCC) }\end{array}$ & $\begin{array}{c}81.65 \text { months } \\
\text { (sD: } 79.3 \text { months) }\end{array}$ \\
\hline HuLme [43] & 14 & 14 & $\begin{array}{l}\text { Secondary/ } \\
\text { tertiary }\end{array}$ & 58.9 & 85.7 & NR & NR & NR & NR & 100 (CRC) & $\begin{array}{c}82.94 \text { months } \\
\text { (sD: } 61.49 \text { months) }\end{array}$ \\
\hline KANG [44] & 447 & 447 & $\begin{array}{l}\text { Secondary/ } \\
\text { tertiary }\end{array}$ & 55.1 & 67.1 & 73.6 & 8.3 & 18.1 & NR & 8.72 (UCC) & $\begin{array}{c}46 \% \text { ( } 2-6 \text { months), } \\
14.09 \% \text { (6-12 months), } \\
21.72 \% \text { ( } 1-5 \text { years), } \\
18.19 \% \text { (>5 years) }\end{array}$ \\
\hline KeLSALL [45] & 62 & 62 & $\begin{array}{c}\text { Secondary/ } \\
\text { tertiary }\end{array}$ & 56.8 & 69.4 & NR & 0.0 & $\mathrm{NR}$ & NR & $\begin{array}{l}32.26 \text { (GORD), } 37.10 \text { (rhinosinusitis), } \\
6.45 \text { (asthma), } 8.06 \text { (EB), } 6.45 \\
\text { (bronchiectasis), } 29.03 \text { (UCC) }\end{array}$ & $\begin{array}{l}42.9 \text { months (IQR: } \\
26-130 \text { months) }\end{array}$ \\
\hline
\end{tabular}




\begin{tabular}{|c|c|c|c|c|c|c|c|c|c|c|c|}
\hline $\begin{array}{l}\text { First author } \\
\text { [ref.] }\end{array}$ & $\begin{array}{l}\text { Total } \\
\text { sample } \\
\text { size }\end{array}$ & $\begin{array}{l}\text { Sample } \\
\text { size of } \\
\text { patients } \\
\text { with } \\
\text { chronic } \\
\text { cough }\end{array}$ & $\begin{array}{l}\text { Type of } \\
\text { care/ } \\
\text { sample } \\
\text { type }\end{array}$ & $\begin{array}{c}\text { Age } \\
\text { (years) }\end{array}$ & $\begin{array}{l}\text { Female } \\
(\%)\end{array}$ & $\begin{array}{l}\text { Never } \\
\text { smoker } \\
(\%)\end{array}$ & $\begin{array}{l}\text { Current } \\
\text { smoker } \\
(\%)\end{array}$ & $\begin{array}{l}\text { Former } \\
\text { smoker } \\
(\%)\end{array}$ & $\begin{array}{l}\text { Smoking } \\
\text { pack history } \\
\text { (pack-years) }\end{array}$ & $\begin{array}{l}\text { Causes of } \\
\text { chronic cough } \\
(\%)\end{array}$ & $\begin{array}{l}\text { Duration of } \\
\text { chronic cough }\end{array}$ \\
\hline KeLSALL [46] & 70 & 70 & $\begin{array}{l}\text { Secondary/ } \\
\text { tertiary }\end{array}$ & 55.0 & 72.9 & 100.0 & 0.0 & $\begin{array}{l}0 \text { (stopped } \\
<6 \text { months) }\end{array}$ & NR & 100 (UCC) & $\begin{array}{l}\text { 62.4 months (IQR: } \\
32.5-131.3 \text { months) }\end{array}$ \\
\hline Koo $[10]^{\S}$ & 401 & 401 & $\begin{array}{l}\text { Secondary/ } \\
\text { tertiary }\end{array}$ & 48.4 & 61.1 & NR & NR & NR & NR & $\begin{array}{c}0 \text { (ILD), } 0 \text { (COPD), } 0 \text { (asthma), } \\
0 \text { (severe bronchiectasis), } \\
0 \text { (lung cancer) }\end{array}$ & NR \\
\hline KosKeLA [47] & 3697 & 976 & $\begin{array}{l}\text { Other; } \\
\text { general } \\
\text { population }\end{array}$ & 47.8 & 82.6 & NR & 6.9 & 31.4 & 6.38 & NR & $\begin{array}{l}57 \% \text { (>13 months), } \\
26 \% \text { (>65 months) }\end{array}$ \\
\hline KoSKELA [48] & 3695 & 975 & $\begin{array}{l}\text { Other; } \\
\text { general } \\
\text { population }\end{array}$ & 47.8 & 82.6 & NR & 31.4 & NR & NR & $\begin{array}{l}10.25 \text { (asthma), } 15.66 \text { (chronic } \\
\text { rhinosinusitis), } 12.59 \text { (GORD) }\end{array}$ & $\begin{array}{l}13.85 \% \text { ( } 2-12 \text { months), } \\
86.15 \% \text { ( } 3-8 \text { weeks) }\end{array}$ \\
\hline KUZnAIR [49] & 136 & 136 & $\begin{array}{l}\text { Secondary/ } \\
\text { tertiary }\end{array}$ & 63.0 & 65.4 & 63.2 & 3.7 & 33.1 & NR & $\begin{array}{c}64.71 \text { (rhinitis or sinusitis), } \\
36.03 \text { (GORD), } 16.18 \text { (asthma), } \\
6.62 \text { (bronchitis), } 6.62 \text { (ILD), } \\
5.88 \text { (postinfectious), } \\
4.41 \text { (bronchiectasis), } 1.47 \text { (ACEi), } \\
6.62 \text { (others) }\end{array}$ & $\begin{array}{l}18 \text { months (IQR: } \\
6-48 \text { months) }\end{array}$ \\
\hline LAI [50] & 1162 & 1162 & $\begin{array}{l}\text { Secondary/ } \\
\text { tertiary }\end{array}$ & 42.6 & 55.4 & 100.0 & 0.0 & 0.0 & NR & $\begin{array}{c}22.29 \text { (EB), } 19.10 \text { (CVA), } \\
12.48 \text { (GORD), } 7.31 \text { (UACS), } \\
38.81 \text { (other) }\end{array}$ & 27.07 months \\
\hline LAN [51] & 164 & 118 & $\begin{array}{l}\text { Secondary/ } \\
\text { tertiary }\end{array}$ & 69.0 & 47.0 & 41.5 & 9.2 & 49.4 & 25.07 & $\begin{array}{c}61.02 \text { (GORD), } 16.95 \text { (ACEi), } \\
12.71 \text { (asthma), } 12.71 \text { (UACS), } \\
9.32 \text { (infection) }\end{array}$ & $\begin{array}{l}89.7 \text { months } \\
\text { (sD: } 123.5 \text { months) }\end{array}$ \\
\hline LANDT [52] & 41545 & 2381 & $\begin{array}{l}\text { Other; } \\
\text { general } \\
\text { population }\end{array}$ & 59.9 & 55.4 & 45.8 & 11.5 & 42.5 & 13.55 & 50.22 (COPD), 49.78 (other) & NR \\
\hline LÄTTI [53] & 3673 & 957 & $\begin{array}{l}\text { Other; } \\
\text { general } \\
\text { population }\end{array}$ & 47.8 & 82.6 & NR & 6.9 & 31.4 & 6.41 & $\begin{array}{l}15.51 \text { (chronic rhinosinusitis), } \\
11.69 \text { (asthma), } 12.59 \text { (GORD) }\end{array}$ & $\begin{array}{c}5.42 \% \text { (<3 weeks), } \\
3.43 \% \text { ( } 3-8 \text { weeks), } \\
7.27 \% \text { (>8 weeks) }\end{array}$ \\
\hline LeE [54] & 49 & 28 & $\begin{array}{l}\text { Secondary/ } \\
\text { tertiary }\end{array}$ & 54.8 & 55.1 & NR & 0.0 & NR & NR & $\begin{array}{l}21 \text { (asthma), } 14 \text { (GORD), } \\
7 \text { (postinfectious disease), } \\
4 \text { (rhinitis), } 4 \text { (EB), } 4 \text { (OSA), } \\
4 \text { (bronchiectasis), } 43 \text { (UCC) }\end{array}$ & 41.5 months \\
\hline LeE [55] & 100 & 100 & $\begin{array}{l}\text { Secondary/ } \\
\text { tertiary }\end{array}$ & 54.0 & 57.0 & 80.0 & 5.0 & 15.0 & NR & $\begin{array}{c}24 \text { (asthma), } 7 \text { (EB), } 9 \text { (rhinitis), } \\
3 \text { (GORD), } 29 \text { (UCC), } 7 \text { (postviral } \\
\text { cough), } 6 \text { (sarcoidosis), } 8 \text { (ILD), } \\
2 \text { (OSA), } 2 \text { (bronchiectasis), } \\
1 \text { (CB), } 1 \text { (ACEi), } 1 \text { (postinfective) }\end{array}$ & 40 months \\
\hline
\end{tabular}




\begin{tabular}{|c|c|c|c|c|c|c|c|c|c|c|c|}
\hline $\begin{array}{l}\text { First author } \\
\text { [ref.] }\end{array}$ & $\begin{array}{l}\text { Total } \\
\text { sample } \\
\text { size }\end{array}$ & $\begin{array}{l}\text { Sample } \\
\text { size of } \\
\text { patients } \\
\text { with } \\
\text { chronic } \\
\text { cough }\end{array}$ & $\begin{array}{l}\text { Type of } \\
\text { care/ } \\
\text { sample } \\
\text { type }\end{array}$ & $\begin{array}{l}\text { Age } \\
\text { (years) }\end{array}$ & $\begin{array}{l}\text { Female } \\
(\%)\end{array}$ & $\begin{array}{l}\text { Never } \\
\text { smoker } \\
(\%)\end{array}$ & $\begin{array}{l}\text { Current } \\
\text { smoker } \\
(\%)\end{array}$ & $\begin{array}{l}\text { Former } \\
\text { smoker } \\
(\%)\end{array}$ & $\begin{array}{l}\text { Smoking } \\
\text { pack history } \\
\text { (pack-years) }\end{array}$ & $\begin{array}{l}\text { Causes of } \\
\text { chronic cough } \\
\text { (\%) }\end{array}$ & $\begin{array}{l}\text { Duration of } \\
\text { chronic cough }\end{array}$ \\
\hline LeE [56] & 32 & 17 & $\begin{array}{l}\text { Secondary/ } \\
\text { tertiary }\end{array}$ & 51.3 & 59.4 & 100.0 & 0.0 & 0.0 & NR & $\begin{array}{c}24 \text { (asthma), } 24 \text { (GORD), } 6 \text { (EB), } \\
6 \text { (post-infectious), } \\
6 \text { (bronchiectasis), } 35 \text { (UCC) }\end{array}$ & $\begin{array}{l}34 \text { months (range: } \\
24-85 \text { months) }\end{array}$ \\
\hline MA [57] & 200 & 110 & $\begin{array}{l}\text { Secondary/ } \\
\text { tertiary }\end{array}$ & 47.0 & 62.0 & 100.0 & 0.0 & 0.0 & NR & $\begin{array}{l}9.09 \text { (UACS), } 49.09 \text { (CVA), } 5.45 \\
\text { (GORD), } 9.09 \text { (NAEB), } 8.18 \text { (post virus } \\
\text { cough), } 9.09 \text { (two causes), } 10 \text { (other) }\end{array}$ & $\begin{array}{l}9 \text { months (range: } \\
2-360 \text { months) }\end{array}$ \\
\hline MeLto [58] & 88 & 88 & $\begin{array}{l}\text { Secondary/ } \\
\text { tertiary }\end{array}$ & 53.1 & 72.7 & 99.4 & NR & NR & NR & $\begin{array}{c}40 \text { (GORD), } 38 \text { (PND), } \\
14 \text { (asthma), } 4 \text { (bronchiectasis), } \\
4 \text { (other) }\end{array}$ & $\begin{array}{l}85.8 \text { months } \\
\text { (sD: } 127.4 \text { months) }\end{array}$ \\
\hline Morice $[59]^{\S}$ & 255 & 185 & $\begin{array}{l}\text { Secondary/ } \\
\text { tertiary }\end{array}$ & 60.8 & 63.1 & NR & NR & NR & NR & NR & NR \\
\hline MURRY [60] & 16 & 16 & NR & $29-69$ & NR & NR & NR & NR & NR & $\begin{array}{l}100 \text { (GORD, laryngopharyngeal } \\
\text { reflux, and concurrent paradoxical } \\
\text { vocal fold movement) }\end{array}$ & $\begin{array}{l}8.4 \text { months } \\
\text { (sD: } 5.3 \text { months) }\end{array}$ \\
\hline Oлоо [61] & 112 & 112 & $\begin{array}{l}\text { Secondary/ } \\
\text { tertiary }\end{array}$ & 56.2 & 65.2 & 53.6 & 5.4 & 41.1 & NR & $\begin{array}{c}29.35 \text { (reflux related cough), } \\
21.74 \text { (asthma), } 11.96 \text { (rhinitis), } \\
4.35 \text { (post-infective), } 11.96 \text { (multiple } \\
\text { causes), } 7.61 \text { (UCC), } 13.04 \text { (other) }\end{array}$ & $\begin{array}{l}39 \text { months (range: } \\
3.25-832 \text { months) }\end{array}$ \\
\hline OzGE [62] & 165 & NR & $\begin{array}{l}\text { Secondary/ } \\
\text { tertiary }\end{array}$ & 43.3 & 58.2 & 41.2 & 38.2 & 20.6 & NR & $\begin{array}{l}25.6 \text { (asthma), } 18 \text { (PND), } \\
21 \text { (sinusitis), } 35.6 \text { (CB) }\end{array}$ & $\begin{array}{l}46.5 \text { months } \\
\text { (sD: } 9.6 \text { months) }\end{array}$ \\
\hline SAто [63] & 129 & 129 & $\begin{array}{l}\text { Secondary/ } \\
\text { tertiary }\end{array}$ & 68.5 & 40.9 & NR & 7.8 & NR & 23.0 & $\begin{array}{c}54.26 \text { (idiopathic interstitial } \\
\text { pneumonias), } 37.98 \text { (connective } \\
\text { tissue disease-associated ILD), } \\
7.75 \text { (chronic hypersensitivity } \\
\text { pneumonitis) }\end{array}$ & NR \\
\hline SHARIAT [64] & 360 & 263 & $\begin{array}{l}\text { Secondary/ } \\
\text { tertiary }\end{array}$ & 39.4 & 100.0 & NR & NR & NR & NR & NR & $\begin{array}{l}260 \text { months of chest } \\
\text { symptoms (range: } \\
6.5-793 \text { months) }\end{array}$ \\
\hline Shembel [65] & 365 & 345 & $\begin{array}{l}\text { Secondary/ } \\
\text { tertiary }\end{array}$ & 53.7 & 84.7 & NR & NR & NR & NR & 100 (UACS) & NR \\
\hline SINHA [66] & 223 & NR & NR & NR & NR & NR & NR & NR & NR & NR & NR \\
\hline SмIтн [67] & 26 & 26 & $\begin{array}{l}\text { Secondary/ } \\
\text { tertiary }\end{array}$ & 68.7 & NR & NR & 0.0 & NR & 42.4 & 100 (COPD) & $\begin{array}{l}117 \text { months (range: } \\
26-936 \text { months) }\end{array}$ \\
\hline SONG [68] & 857 & 37 & $\begin{array}{l}\text { Other; } \\
\text { general } \\
\text { population }\end{array}$ & 76.8 & 57.0 & 62.2 & 12.2 & 25.5 & NR & $\begin{array}{c}16.2 \text { (asthma), } 13.5 \text { (allergic rhinitis), } \\
2.8 \text { (GORD) }\end{array}$ & NR \\
\hline $\begin{array}{l}\text { TERnESTEN- } \\
\text { HASSEUS [69] }\end{array}$ & 121 & 62 & $\begin{array}{l}\text { Secondary/ } \\
\text { tertiary }\end{array}$ & 53.6 & 64.5 & 61.3 & 0.0 & 38.7 & NR & 100 (UCC) & $\begin{array}{l}137.8 \text { months } \\
\text { (sD: } 130 \text { months) }\end{array}$ \\
\hline
\end{tabular}




\begin{tabular}{|c|c|c|c|c|c|c|c|c|c|c|c|}
\hline $\begin{array}{l}\text { First author } \\
\text { [ref.] }\end{array}$ & $\begin{array}{l}\text { Total } \\
\text { sample } \\
\text { size }\end{array}$ & $\begin{array}{l}\text { Sample } \\
\text { size of } \\
\text { patients } \\
\text { with } \\
\text { chronic } \\
\text { cough }\end{array}$ & $\begin{array}{l}\text { Type of } \\
\text { care/ } \\
\text { sample } \\
\text { type }\end{array}$ & $\begin{array}{l}\text { Age } \\
\text { (years) }\end{array}$ & $\begin{array}{l}\text { Female } \\
(\%)\end{array}$ & $\begin{array}{l}\text { Never } \\
\text { smoker } \\
(\%)\end{array}$ & $\begin{array}{l}\text { Current } \\
\text { smoker } \\
(\%)\end{array}$ & $\begin{array}{l}\text { Former } \\
\text { smoker } \\
(\%)\end{array}$ & $\begin{array}{c}\text { Smoking } \\
\text { pack history } \\
\text { (pack-years) }\end{array}$ & $\begin{array}{l}\text { Causes of } \\
\text { chronic cough } \\
\text { (\%) }\end{array}$ & $\begin{array}{l}\text { Duration of } \\
\text { chronic cough }\end{array}$ \\
\hline $\begin{array}{l}\text { VAN DE KERKHOVE } \\
{[70]}\end{array}$ & 51 & 51 & $\begin{array}{l}\text { Secondary/ } \\
\text { tertiary }\end{array}$ & NA & 80.4 & NR & NR & NR & NR & 100 (UCC) & $\begin{array}{l}48 \text { months (IQR: } \\
2-192 \text { months) }\end{array}$ \\
\hline VAZquez [71] & 1422 & 552 & NR & 54.8 & 76.6 & NR & 61.7 & NR & 37.59 & 100 (COPD) & $\begin{array}{c}28.13 \% \text { ( } 3 \text { months to } \\
2 \text { years), } 10.69 \% \\
(>2 \text { years) }\end{array}$ \\
\hline VERNON [16] & 22 & 22 & $\begin{array}{l}\text { Secondary/ } \\
\text { tertiary }\end{array}$ & 66.1 & 72.7 & NR & NR & NR & NR & $\begin{array}{c}31.82 \text { (GORD), } 22.73 \text { (asthma), } \\
18.18 \text { (bronchitis), } 18.18 \text { (PND), } \\
\text { irritant exposure, post-infectious } \\
\text { cough, mild COPD }\end{array}$ & $\begin{array}{c}100 \% \text { (>8 weeks), } \\
81.8 \% \text { (>52 weeks) }\end{array}$ \\
\hline VERNON [15] & 39 & 24 & Primary & 44.1 & 71.8 & NR & 0.0 & NR & NR & $\begin{array}{c}20.83 \text { (asthma), } 20.83 \text { (GORD), } \\
8.33 \text { (irritable larynx), } 12.5 \text { (irritant } \\
\text { exposure), } 25 \text { (PND), } \\
8.33 \text { (other), } 50 \text { (unknown) }\end{array}$ & $\begin{array}{c}38.46 \% \text { ( } 3 \text { weeks to } \\
2 \text { months), } \\
17.95 \% \text { ( } 2-6 \text { months), } \\
10.26 \% \text { (6-12 months), } \\
30.77 \% \text { ( }>1 \text { year) }\end{array}$ \\
\hline VERTIGAN $[72]^{\S}$ & 38 & 11 & $\begin{array}{l}\text { Secondary/ } \\
\text { tertiary }\end{array}$ & 56.1 & 77.0 & NR & 11.0 & NR & NR & $\begin{array}{c}49 \text { (reflux), } 29 \text { (asthma), } \\
17 \text { (rhinitis) }\end{array}$ & NR \\
\hline VERTIGAN [73] & 171 & 111 & $\begin{array}{l}\text { Secondary/ } \\
\text { tertiary }\end{array}$ & 53.3 & 76.6 & NR & NR & NR & NR & $\begin{array}{c}50.45 \text { (CRC), } 49.55 \text { (CRC/paradoxical } \\
\text { vocal fold movement) }\end{array}$ & NR \\
\hline VERTIGAN [74] & 171 & 111 & $\begin{array}{l}\text { Secondary/ } \\
\text { tertiary }\end{array}$ & 56.4 & 76.6 & NR & 3.5 & NR & NR & $\begin{array}{l}55 \text { (GORD), } 18 \text { (asthma), } \\
49 \text { (PND), } 35 \text { (allergies) }\end{array}$ & NR \\
\hline VERTIGAN [75] & 53 & 53 & $\begin{array}{l}\text { Secondary/ } \\
\text { tertiary }\end{array}$ & 60.0 & 66.0 & 100.0 & 0.0 & 0.0 & NR & $100(\mathrm{CRC})$ & 59 months \\
\hline Won [76] & 478 & 478 & $\begin{array}{l}\text { Secondary/ } \\
\text { tertiary }\end{array}$ & 55.1 & 68.4 & 74.7 & 7.7 & 17.7 & NR & $\begin{array}{c}12.97 \text { (UCC), } 87.03 \text { (chronic cough } \\
\text { with unspecified underlying } \\
\text { condition) }\end{array}$ & $\begin{array}{l}44 \% \text { (8-24 weeks), } \\
13.8 \% \text { ( } 24-48 \text { weeks), } \\
21.6 \% \text { (52-269 weeks), } \\
20.6 \% \text { ( }>260 \text { weeks) }\end{array}$ \\
\hline $\mathrm{Xu}[77]$ & 87 & 87 & $\begin{array}{l}\text { Secondary/ } \\
\text { tertiary }\end{array}$ & 45.1 & 55.2 & NR & NR & NR & NR & 100 (GORD) & $\begin{array}{l}8.28 \text { months } \\
\text { (sD: } 30.83 \text { months) }\end{array}$ \\
\hline ZHAN [11]" & 237 & 237 & $\begin{array}{c}\text { Secondary/ } \\
\text { tertiary }\end{array}$ & 40.4 & 54.0 & NR & NR & NR & NR & NR & $\begin{array}{c}43 \text { months } \\
\text { (sD: } 77.3 \text { months) }\end{array}$ \\
\hline
\end{tabular}




\section{Concept elicitation from included studies}

Through iterative discussion of the taxonomy with the steering group, both the urge-to-cough sensation (noted in 31 studies) and the cough symptom itself (noted in 58 studies) emerged as broad domains of cough severity under which eligible items were classified (table 3 and figure 2).

\section{Urge-to-cough sensation}

Regarding the urge-to-cough sensation, we identified two subdomains that mediate its impact: urge frequency $(n=5)$ and intensity $(n=6)$ (table 3 and figure 2). Urge-to-cough sensations, commonly described as an "irritation" $(n=8)$ or "tickle" $(n=9)$ within the throat or chest region, can precede a cough. Patients who experience urge-to-cough sensations frequently report that the urge almost always leads to coughing, although not all coughs are preceded by an urge [42]. Patients described the urge-to-cough as being linked to how frequently one coughs and whether one is able to control their cough: "Well I think the more you try and like think 'Don't let the tickle ... don't let the tickle bother you and don't cough', sometimes the tickle comes like to a point where you just can't stop it” [43]. Thus, the frequency and intensity of urge-to-cough sensations may be related to the subjective experience of coughing and therefore relevant in assessing cough severity. Both urge frequency and intensity were characterised as single-item subdomains (table 3).

\section{Cough symptoms}

The study team identified five subdomains for assessing severity of the cough symptom itself: control $(n=10)$, frequency $(n=33)$, fit/bout duration $(n=4)$, intensity $(n=17)$, and associated features/sequelae $(n=44)$ (table 3 and figure 2).

Cough control refers to the ability, or lack thereof, to voluntarily or involuntarily suppress a cough from starting or once started. Because it captured whether coughing was a conscious act or an uncontrollable, involuntary reflex, the study team characterised cough control as a separate subdomain from cough frequency (table 3 and figure 2). Some patients described voluntarily coughing to clear mucus, "something stuck", or in response to an irritating urge in the throat; other patients described uncontrollable bouts of coughing that are highly unpleasant. Suppression of cough from starting $(n=3)$ or once started $(n=3)$ represented two items under the domain cough control that the study team identified.

Cough frequency represented another subdomain in assessing cough severity (table 3 and figure 2). In some studies, cough frequency referred to the frequency of any coughs (individual or bouts of coughing; $n=28)$; other studies referred to the frequency of bouts $(n=12)$. Suggested periods to assess cough frequency included overall (throughout the day and night; $n=28)$, during the day $(n=14)$, during the night $(n=21)$, in the morning $(n=6)$, and prandial or postprandial $(n=8)$. Studies identified that the frequency of coughing may be variable depending on the time of day (i.e. less frequent at night versus during the day), and that timing dictated various cough impacts to quality of life, such as daytime activities or sleep disruption. Thus, the study team captured six candidate items under the domain frequency: 1) frequency of any coughs (assessed day and night); 2) frequency of coughs during the day; 3) frequency of coughs during the night; 4) frequency of coughs in the morning; 5) frequency of coughs in the prandial/ postprandial period; and 6) frequency of bouts.

The study team identified duration of bouts as a subdomain of cough severity (table 3 and figure 2). The team defined a bout as a prolonged series of explosive phases of coughing, either after a single breath or with several breaths interspersed [79]. Patients may describe greater cough severity when they occur in bouts, potentially as they are "less controllable" and involve greater amounts of "effort”: “... [a bout is] one of those that goes on and on and on and I think 'I can't do with that, it's tired me out' ... you know drained” [43]. Further, longer bouts of coughing may lead to a higher frequency and intensity of adverse sequelae: "To me that strikes me as always a very serious bout of coughing, because obviously, oxygen wasn't getting where it was supposed to be getting. Um, and I find this is freaky, especially if you're driving, you know, and you're coughing, and all of a sudden you're dizzy" [16]. Therefore, the frequency of bouts, along with the duration of each bout, is likely to influence cough severity. The team characterised duration of bouts as a single-item subdomain.

The study team identified cough intensity as another subdomain (table 3 and figure 2). Single-word descriptions of cough intensity provided seven items under this subdomain, including harsh $(\mathrm{n}=2)$, deep $(n=2)$, hacking $(n=2)$, barking $(n=1)$, honking $(n=1)$, strong $(n=2)$, and intense $(n=1)$. The team also classified patients' description of the effort to cough $(n=6)$, defined as the physical effort to expectorate an urge, as another item under intensity. 
TABLE 3 Domains and items related to measurement of cough severity in adult patients with chronic cough

Domain, $\mathrm{n} \quad$ Subdomain, $\mathrm{n} \quad$ Item, $\mathrm{n} \quad$ Example phrases

Urge-to-cough $(n=31)$ : sensations (i.e.

laryngeal, chest) that

can lead to cough

"Although technically not a "cough" as defined medically, patients considered this sensation (urge) an inseparable part of the cough experience indicating it should be a component of an evaluation tool."

Urge frequency Urge frequency "The most common descriptions were abnormal sensation in the throat, phlegm and mucus in the throat, tickle and

irritation in the throat and a tickle in the throat. The median response for abnormal sensation, phlegm and irritation was 3.0 [on a scale of $1=$ all of the time to $7=$ none of the time] which indicates that the majority of patients rated that item occurring at least a good bit of the time."

"There was infrequent occurrence of pushing on the chest, pressing on the throat, food catching, itch and tingle. The majority of these participants rated these items between 4.0 and 7.0 ranging from some of the time to none of the time." "How much warning do you get before you cough?

$1=$ None, $2=$ Occasionally, 3=Frequently"

"Meanwhile, a study of urge to cough and associated somatic sensations in 100 unselected patients with chronic cough attending a specialist clinic in UK found that $91 \%$ always cough in response to urge to cough and that the urge to cough was frequently associated with abnormal throat sensations such as irritation (86\%) and tickling (73\%)."

Urge intensity Urge intensity "Sometimes I can't sleep at all night. I have to get up and go get in a chair, because whatever is coming out of my head or

my throat is sitting in this bronchial tube down there, and it's messing up everything."

"In the last 2 weeks...I have been annoyed by an irritation, such as a tickle or itch in my throat"

"Well I think the more you try and like think 'Don't let the tickle ... don't let the tickle bother you and don't cough', sometimes the tickle comes like to a point where you just can't stop it."

"People with chronic cough may cough deliberately in response to a sensation in the throat even though referred for behavioural management of cough. The issue, in these cases, is the perception of a throat sensation rather than the actual cough."

"And that then trickles down here, and then I cough like the devil... I get-it sticks here, great big chunks of it will stick here-can't get it out."

"Congestion in throat (as though something "fuzzy" there that needs to be swallowed) and chest. Need to clear throat to get rid of it."

$\begin{array}{cc}\text { Cough }(n=58) & \begin{array}{c}\text { Cough control to } \\ (n=10)\end{array} \\ & \begin{array}{c}\text { prevent a cough } \\ \text { from starting }\end{array}\end{array}$

$(n=3)$

"People with chronic cough may cough deliberately in response to a sensation in the throat even though referred for behavioural management of cough."

"It is hypothesised that warning before the cough characterised by irritation in the throat or a strangulating sensation is an important precursor to controlling the cough and that if people can anticipate that a cough is about to occur they can be taught strategies to inhibit the cough before it starts."

"Do you cough deliberately to clear something from your throat? 1=None, 2=Occasionally, 3=Frequently"

Cough control "The majority of respondents (63\%) were unable to suppress their cough."

once a cough has "The final cough was absolutely - absolutely retched and, ugh, I think was XXXXX who mentioned embarrassed. Ugh, I had a started $(n=3)$ lot of - I had a number of meetings that I - that I had to go to and, uh, I couldn't stop coughing in those things."

"An uncontrolled cough is very unpleasant for the patient."

"The cough controlled me, I had no control at all over when I coughed, how often I coughed, how strong the cough was ... it got to the point where ... I didn't even want to go out to get my shopping." 
TABLE 3 Continued

Subdomain, $\mathbf{n}$

Item, n

Example phrases

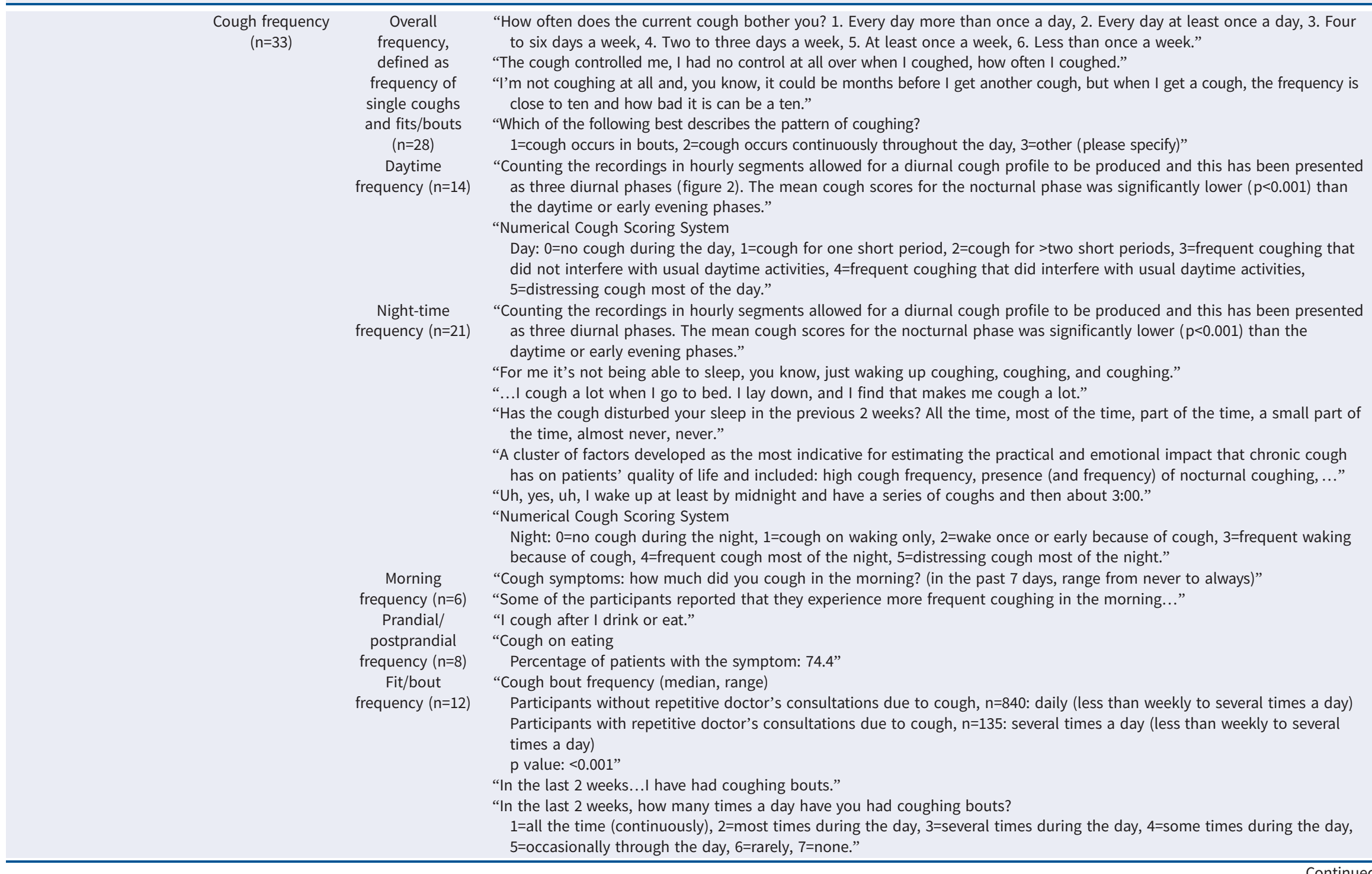


TABLE 3 Continued

Domain, $\mathrm{n}$

Subdomain, $\mathrm{n}$

Item, $\mathrm{n}$

Example phrases

Fit/bout duration Fit/bout duration "Paroxysms of coughing, which were extended bouts of individual coughs and that were described as less controllable than

a single cough, were also a component of frequency."

"Quantification in terms of epochs (frequency) effectively ignored epoch length; the poorer correlations with cough quality of life and VAS imply that epoch length was important to patients."

Intensity $(n=17)$

"Patients' perceptions of the severity of their cough remain important and may not correlate [with cough frequency] for a number of reasons, including changes in cough intensity."

Effort to cough "It sticks here, great big chunks of it will stick here-can't get it out. And I cough, and cough, and cough, and then if you go $(n=6)$

to a restaurant and start coughing they throw you out"

"Difficulty to expectorate."

"Sputum symptoms: how difficult was it for you to bring up phlegm? (in the past 7 days, ranging from "not at all" to "a lot/ extremely")"

"In the last 2 weeks...I have found it difficult to cough up phlegm (sputum)"

"This physical effort [of coughing] was associated with a range of [adverse] symptoms."

"Patients described the physicality of the cough and described the sheer effort a 'fit' took."

Harshness of

cough ( $n=2)$

Deepness of

cough $(n=2)$

Hacking due to

cough $(n=2)$

Barking due to

cough $(n=1)$

Honking due to

cough ( $n=1$ )

Strong cough $(n=2)$

Intense cough

$(n=1)$

"Intensity of coughing was described in terms of how 'deep,' 'hard,' or 'harsh,' the coughing was."

“...it was a really deep hacking, not just an ordinary sort of cough, very deep, you know, from your chest.”

"Mine is very hacking, and it's not intense or deep, and nothing comes up. It's not productive.... It's dry hacking."

"Character of cough: Barking - having a sound quality similar to that of a dog's bark."

"Character of cough: Honking - sounding like the honk of a Canadian wild goose."

“I had no control at all over when I coughed, how often I coughed, how strong the cough was."

"Mine is very hacking, and it's not intense or deep."

"I have it under control with the medication, but I still get some fits that usually last five minutes, and they're rather intense, uh, in coughing."

\section{Experiences while coughing that are associated with intensity}

Associated features/sequelae

$(n=44)$

cough $(n=4)$

Syncope due to

cough $(n=6)$

Wheeze due to

cough $(n=13)$

Dyspnoea/

shortness of

breath due to "To me that strikes me as always a very serious bout of coughing, because obviously, oxygen wasn't getting where it was
supposed to be getting. Um, and I find this is freaky, especially if you're driving, you know, and your coughing, and all of a sudden you're dizzy.'

cough $(n=15)$

"I feel I may pass out because of my coughing."

"Cough was commonly associated with other symptoms such as $[\ldots]$ wheeze $(37 \%) \ldots$...

"Cough was commonly associated with other symptoms such as breathlessness (55\%)..."

"This physical effort was associated with a range of symptoms [...]: It's retching, it's struggling for breath [...] It's like sometimes it's working its way up to a crescendo until I am sick." 
TABLE 3 Continued

Domain, $\mathrm{n}$

Subdomain, $n$

Item, $\mathrm{n}$

Example phrases

Incontinence due "In addition, cough resulted in disturbed sleep in 70\%, sore throat in $45 \%$ and caused incontinence in $55 \%$ of women and

to cough $(n=15) \quad 5 \%$ of men.”

"Incontinence

No problem: 77 (57\% of chronic cough patients)

Small problem: 41 (30\% of chronic cough patients)

Major problem: 18 (13\% of chronic cough patients)"

"In the last 2 weeks...I have been incontinent of urine (wet my underwear) during coughing."

"Sometimes I wet on myself I cough so hard and it makes my head hurt."

"[He] coughed so hard that [...] now, I have incontinence [sic] so bad, that I have to urinate every hour."

"[Cough] was the most important symptom in $31 \%$ of patients and up to $36 \%$ had reached the stage of retching, vomiting or

being incontinent during cough episodes."

Tightness of

throat due to

cough $(n=4)$

Tightness of

chest due to

cough $(n=5)$

Distress due to

cough $(n=3)$

"I feel tightness in my throat when I am having a coughing problem."

"Within the last month, how did the following problems affect you? ( $0=$ no problem and $5=$ severe/frequent problem) Chest tightness when coughing"

"Finally, several studies reviewed included measures that assessed intensity of cough (e.g. distressing cough; chest/ abdominal pain)."

Immediate experiences after coughing that are associated with intensity depending on susceptibility

General pain due "I've gotten to that point where I'm coughing, I mean, the diaphragm, the rib cage, umm, is painful."

to cough $(n=6) \quad$ "Have you had chest or stomach pain due to cough in the last 2 weeks? All the time, most of the time, part of the time, a

Rib pain or small part of the time, almost never, never."

fracture due to "What are the following symptoms or ailments have you had in the last month? Chest pain during exertion, [...] back pain,

cough $(n=6)$

Chest pain due

to cough $(n=13)$

Abdominal pain

due to cough

$(n=6)$

...”

"Broken ribs, retching, chest pains, or hoarseness

No problem: 64 (47\% of chronic cough patients)

Small problem: 47 (35\% of chronic cough patients)

Major problem: 25 (18\% of chronic cough patients)"

"In the last 2 weeks...I have had chest pain during coughing."

Back pain due to "[He] coughed so hard that I have developed costacondritis [sic] (cracked rib cartilage) twice and ended up in the emergency

cough $(n=2)$

room, adding that now, I have incontinence [sic] so bad, that I have to urinate every hour."

"Last year the cough was so severe I fractured two ribs."

"I am at my wits end," adding "my ribs and my back are very sore from all the coughing."

Aches due to "Spectrum and frequency of adverse occurrences before and after treatment in patients whose cough was eliminated

cough $(n=1) \quad$ Achiness: before treatment (\%): 21; after treatment (\%): 0 "

Headache due to

cough $(n=8)$

"Have you had stomach pain or headache as a result of cough? (not at all to extremely)"

"Sometimes I throw up a lot of mucus and I really cough a lot at night... Sometimes I wet on myself I cough so hard and it makes my head hurt."

"However transient, severe headache upon coughing [...] is defined as "cough headache". Cough headache [is] considered to be a form of exertional headache." 


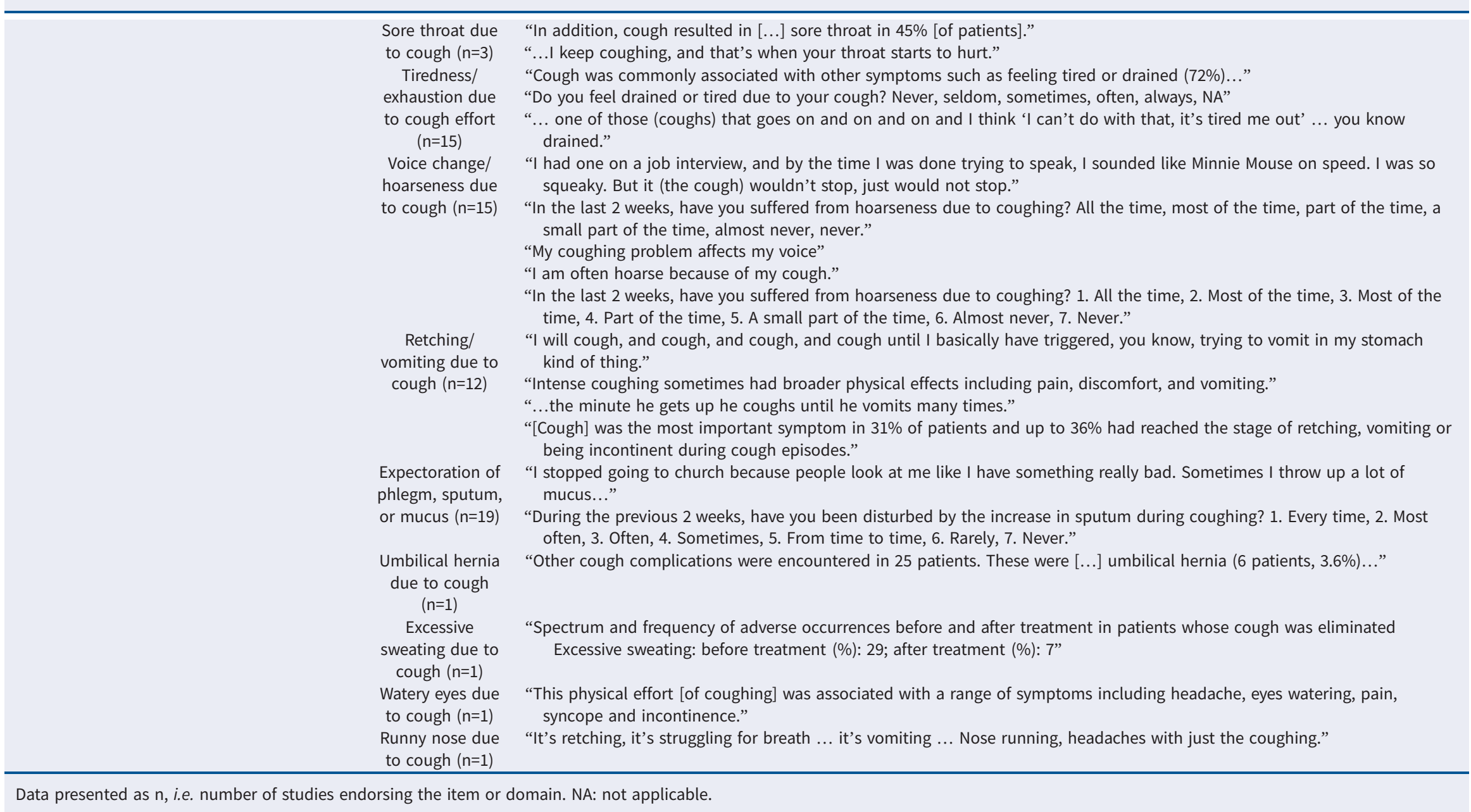




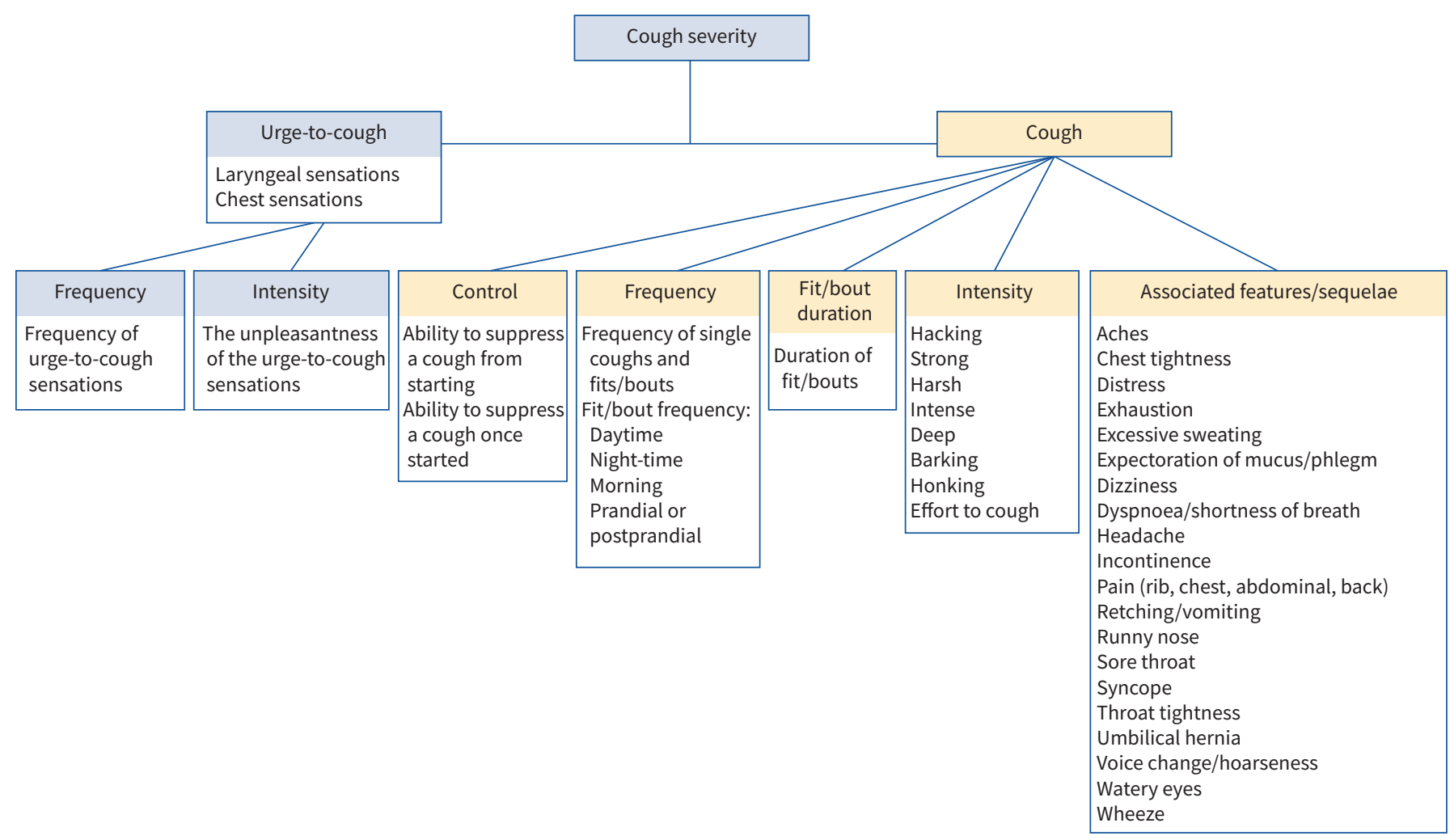

FIGURE 2 Conceptual framework of cough severity in patients with chronic cough.

Related to intensity, patients described symptoms that they experience during and immediately after coughing. The study team classified this as a separate subdomain called associated features/sequalae of coughing (table 3 and figure 2). Under this subdomain, eight items representing experiences while coughing included dizziness $(n=4)$, wheeze $(n=13)$, syncope $(n=6)$, dyspnoea or shortness of breath $(n=15)$, incontinence $(n=15)$, distress $(n=3)$, tightness of throat $(n=4)$, and tightness of chest $(n=5)$. The team also identified sequalae of coughing, depending on patient susceptibility, as 16 other items under this subdomain, including general pain $(n=6)$, rib pain or fracture $(n=6)$, chest pain $(n=13)$, abdominal pain $(n=6)$, back pain $(n=2)$, aches $(n=1)$, headache $(n=8)$, sore throat $(n=3)$, exhaustion $(n=15)$, voice change or hoarseness $(n=15)$, retching or vomiting $(n=12)$, expectoration of mucus or phlegm $(n=19)$, umbilical hernia $(n=1)$, excessive sweating $(n=1)$, watery eyes $(n=1)$, and runny nose $(n=1)$.

\section{Discussion}

We report the first systematic survey synthesising items and domains of cough severity in patients with refractory or unexplained chronic cough. The survey identified 82 potential items, of which 43 proved unique and clearly related to cough severity. The study team categorised these final items under two broad domains: the urge-to-cough sensation (two subdomains: urge frequency and intensity) and the cough symptom itself (five subdomains: cough control, frequency, duration of bouts, intensity, and associated features/sequelae).

\section{Strengths and limitations}

Our work follows established methodology and guidance for optimal development of PROMs [20]. A preliminary step in PROM development is to establish the measurement construct, a theoretically derived notion of the domain(s) to be measured [18-20]. An understanding of the construct will lead to expectations about how an instrument should behave if it is valid [19]. Therefore, the systematic survey and conceptual framework in itself represent strengths in terms of development of a cough severity PROM.

Other strengths of our systematic survey include: 1) transparent eligibility criteria and rigorous methods for systematic data abstraction; 2) flexibility in development of the taxonomy allowing incorporation of the views of involved reviewers while abstracting; and 3) achieving a saturation effect (i.e. few new items/ 
domains) after abstraction of approximately 20 eligible studies. It is therefore unlikely that we missed any key items or domains. Inclusion of studies from different geographic and care settings ensured that items and domains are likely to reflect experiences from a diversity of chronic cough patients. Furthermore, development of our conceptual framework involved iterative discussion with a steering group of chronic cough and health status measurement experts, thereby strengthening the clarity and face validity of our framework.

Our survey, however, has limitations. The database search primarily focused on studies from the year 2007 onwards, although articles published before 2007 were still hand-searched and included if eligible. The focus on more recent studies was based on developing conceptual insights relying on current, rather than historical, patient experiences of cough severity. Because the study achieved saturation in the taxonomy, a more exhaustive search would unlikely change the results.

The process of synthesising verbatim quotations to characterise items and domains introduced subjectivity, as did the decisions regarding combining, reducing and separating of items. For example, a number of statements addressed frequency of coughing during different periods of the day, and we labelled these items as daytime frequency, night-time frequency, morning frequency and prandial/postprandial frequency. Others may have classified these into fewer or greater items.

\section{Findings in context of other studies}

A study by VERNON et al. [16] explored factors relevant to assessment of cough severity in a literature review and a semi-structured focus group study. Their conceptual framework consisted of frequency, intensity and disruption, and informed development of the CSD [15]. Our results are consistent with several of the themes presented in their conceptual framework, although our survey identified additional important concepts. Firstly, we identified both the frequency and intensity of the urge-to-cough sensation as subdomains that should be assessed in measuring cough severity. The conceptual framework of the CSD includes an item related to urge frequency, although assessment of urge intensity is not considered.

Secondly, our conceptual framework identifies cough control as a subdomain. Recent mechanistic studies demonstrate that patients with refractory chronic cough have impaired ability to voluntarily suppress coughing [80], but also provide evidence of impairment in the inhibitory control neurons that represent a sub-conscious and involuntary process [81]. This distinction may be mechanistically relevant. Lack of sub-conscious inhibitory control pathways intuitively seems more important in starting a cough, given that healthy people do not consciously suppress their coughing throughout the day. In contrast, once a cough has started, impaired voluntary suppression may perpetuate longer durations and bouts of coughing.

Functional brain magnetic resonance imaging also corroborates the relevance of central cortical pathways in the conscious and sub-conscious control of coughing [82-84]. As speech therapy aims to increase control of cough, the concept seems highly relevant in assessing the effectiveness of therapies. The conceptual framework of the CSD does not identify this as a stand-alone concept, and few other cough-specific PROMs have included cough control as a domain.

Thirdly, although we identified this as potentially relevant, the CSD does not consider duration of bouts in their conceptual framework. In a study comparing objective and subjective measurements of cough, investigators reported moderate correlation of frequency of cough epochs (bouts) with subjective quality of life and cough severity VAS scores [46]. As the frequency of coughing bouts ignores the length of each bout, the study suggested that duration of bouts may be an important factor in the perception of cough severity that remains unaccounted for with objective bout frequency. Therefore, we have included duration of bouts as a subdomain in our conceptual framework.

Although the CSD framework includes cough intensity, their conceptual framework is non-specific in terms of the subcategories that comprise this domain. The authors identify harshness and physical discomfort as comprising cough intensity. Our survey, however, identified 32 items describing intensity, including the effort to cough, descriptors of coughing (i.e. strong, harsh, intense, deep, barking, honking), and associated features/sequalae (i.e. chest pain, syncope, dizziness, hoarseness, sore throat) (figure 2).

Finally, the CSD framework identifies disruption to daytime activities and sleep as concepts of cough severity. We strove for conceptual clarity by excluding domains or items related to cough impact on quality of life. Several validated and widely available PROMs already assess the impact of cough symptoms on quality of life, including the LCQ [7], CCIQ [8], CQLQ [9], COAT [10], and CET [11]. Our 
conceptual framework defines concepts to measure symptom severity, which would inform an instrument complementary to existing quality of life questionnaires.

\section{Conclusions}

We report a systematic survey and conceptual framework that identified candidate items and domains to measure cough severity. Studies provided 43 unique items that contributed to our conceptual framework. The urge-to-cough sensations, a linked component of the cough experience, classified as a domain. The study team identified frequency (1 item) and intensity (1 item) as subdomains under the urge-tocough sensation. The cough symptom itself represented another domain, subdomains of which included control (2 items), frequency (6 items), fit/bout duration (1 item), intensity (8 items) and associated features/ sequelae (24 items). Qualitative studies could address the comprehensiveness and appropriateness of the items, domains, and subdomains we have identified, and lead to refinement of our evolving approach to measurement of cough severity. These studies, including focus groups with chronic cough patients and consultation with international cough experts, are currently underway. Our findings support item generation and content validity of a PROM assessing cough symptom severity in patients with refractory or unexplained chronic cough. The PROM will be designed for both discriminative and evaluative purposes for use in health research and in clinical practice.

Provenance: Submitted article, peer reviewed.

Author contributions: E. Kum, G.H. Guyatt, T. Devji, Y. Wang, P.M. O'Byrne, and I. Satia conceived the study. E. Kum, G.H. Guyatt, and I. Satia comprised the steering group. E. Kum and R. Couban developed the search strategy. E. Kum, L. Bakaa, L. Lan, E. Liu, A. Mastrolonardo, and R. Couban searched and extracted the data. E. Kum performed the analysis. E. Kum, G.H. Guyatt, and I. Satia interpreted the data. E. Kum drafted the manuscript. All authors critically reviewed and approved the final manuscript.

Conflict of interest: E. Kum has nothing to disclose. G.H. Guyatt has nothing to disclose. T. Devji has nothing to disclose. Y. Wang has nothing to disclose. L. Bakaa has nothing to disclose. L. Lan has nothing to disclose. E. Liu has nothing to disclose. A. Mastrolonardo has nothing to disclose. R. Couban has nothing to disclose. P.M. O'Byrne reports grants and personal fees from AstraZeneca and Medimmune, personal fees from GSK and Chiesi, and grants from Novartis, outside the submitted work. I. Satia reports personal fees from Educational Talks for GPs, GSK and AstraZeneca, grants from ERS Respire 3 Marie Curie Fellowship and E. J. Moran Campbell Early Career Award, and grants and personal fees from Merck Canada, outside the submitted work.

Support statement: E. Kum is supported by a CGS-M Scholarship from the Canadian Institutes of Health Research. I. Satia is supported by an E. J. Moran Campbell Early Career Award from the Department of Medicine, McMaster University (Hamilton, ON, Canada).

\section{References}

$1 \quad$ Song WJ, Chang YS, Faruqi S, et al. The global epidemiology of chronic cough in adults: a systematic review and meta-analysis. Eur Respir J 2015; 45: 1479-1481.

2 Gibson P, Wang G, McGarvey L, et al. Treatment of unexplained chronic cough: CHEST guideline and expert panel report. Chest 2016; 149: 27-44.

3 McGarvey L, Birring S, Morice A, et al. Two Phase 3 Randomized Clinical Trials of Gefapixant, a P2X3 Receptor Antagonist, in Refractory or Unexplained Chronic Cough (COUGH-1 and COUGH-2). Eur Respir J 2020; 56: Suppl. 64, 3800.

4 Morice AH, Birring SS, Smith JA, et al. Characterization of patients with refractory or unexplained chronic cough participating in a phase 2 clinical trial of the P2X3-receptor antagonist gefapixant. Lung 2021; 199: 121-129.

$5 \quad$ Birring SS, Spinou A. How best to measure cough clinically. Curr Opin Pharmacol 2015; 22: 37-40.

6 Higgins JP, Thomas J, Chandler J, et al., eds. Cochrane Handbook for Systematic Reviews of Interventions version. 2nd Edn. Chichester, John Wiley \& Sons, 2019.

7 Birring SS, Prudon B, Carr AJ, et al. Development of a symptom specific health status measure for patients with chronic cough: Leicester Cough Questionnaire (LCQ). Thorax 2003; 58: 339-343.

8 Baiardini I, Braido F, Fassio O, et al. A new tool to assess and monitor the burden of chronic cough on quality of life: Chronic Cough Impact Questionnaire. Allergy 2005; 60: 482-488.

9 French CT, Irwin RS, Fletcher KE, et al. Evaluation of a cough-specific quality-of-life questionnaire. Chest 2002; 121: 1123-1131.

10 Koo HK, Jeong I, Kim JH, et al. Development and validation of the COugh Assessment Test (COAT). Respirology 2019; 24: 551-557. 
11 Zhan W, Zhang L, Jiang M, et al. A new simple score of chronic cough: cough evaluation test. BMC Pulm Med 2020; 20: 68.

12 Boulet LP, Coeytaux RR, McCrory DC, et al. Tools for assessing outcomes in studies of chronic cough: CHEST guideline and expert panel report. Chest 2015; 147: 804-814.

13 Streiner DL, Norman GR, Cairney J. Health Measurement Scales: A Practical Guide to their Development and Use. Oxford, Oxford University Press, 2015.

14 Hsu J, Stone R, Logan-Sinclair R, et al. Coughing frequency in patients with persistent cough: assessment using a 24 hour ambulatory recorder. Eur Respir J 1994; 7: 1246-1253.

15 Vernon M, Kline Leidy N, Nacson A, et al. Measuring cough severity: development and pilot testing of a new seven-item cough severity patient-reported outcome measure. Ther Adv Respir Dis 2010; 4: 199-208.

16 Vernon M, Leidy NK, Nacson A, et al. Measuring cough severity: perspectives from the literature and from patients with chronic cough. Cough 2009; 5: 5.

17 Martin Nguyen A, Bacci E, Dicpinigaitis P, et al. Quantitative measurement properties and score interpretation of the Cough Severity Diary in patients with chronic cough. Ther Adv Respir Dis 2020; 14: 1753466620915155.

18 Guyatt GH, Bombardier C, Tugwell PX. Measuring disease-specific quality of life in clinical trials. CMAJ 1986; 134: 889-895.

19 Guyatt GH, Feeny DH, Patrick DL. Measuring health-related quality of life. Ann Intern Med 1993; 118: 622-629.

20 Kirshner B, Guyatt G. A methodological framework for assessing health indices. J Chronic Dis 1985; 38: 27-36.

21 Moher D, Liberati A, Tetzlaff J, et al. Preferred reporting items for systematic reviews and meta-analyses: the PRISMA statement. BMJ 2009; 339: b2535.

22 Irwin RS, French $\mathrm{CL}$, Chang $\mathrm{AB}$, et al. Classification of cough as a symptom in adults and management algorithms: CHEST Guideline and Expert Panel Report. Chest 2018; 153: 196-209.

23 Thomas J, Harden A. Methods for the thematic synthesis of qualitative research in systematic reviews. BMC Med Res Methodol 2008; 8: 45.

24 Adams RJ, Appleton SL, Wilson DH, et al. Associations of physical and mental health problems with chronic cough in a representative population cohort. Cough 2009; 5: 10.

25 Campi G, Noale M, Fabbrizzi A, et al. The demographic and clinical characteristics of an Italian population of adult outpatients with chronic cough. Aging Clin Exp Res 2020; 32: 741-746.

26 Chamberlain SA, Garrod R, Douiri A, et al. The impact of chronic cough: a cross-sectional European survey. Lung 2015; 193: 401-408.

27 Chan KK, Ing AJ, Laks L, et al. Chronic cough in patients with sleep-disordered breathing. Eur Respir J 2010; 35: $368-372$.

28 Cheng JZ, Wilcox PG, Glaspole I, et al. Cough is less common and less severe in systemic sclerosis-associated interstitial lung disease compared to other fibrotic interstitial lung diseases. Respirology 2017; 22: 1592-1597.

29 Colak Y, Afzal S, Lange P, et al. Role and impact of chronic cough in individuals with asthma from the general population. J Allergy Clin Immunol Pract 2019; 7: 1783-1792.

30 Colak Y, Nordestgaard BG, Laursen LC, et al. Risk factors for chronic cough among 14669 individuals from the general population. Chest 2017; 152: 563-573.

31 Cornford CS. Why patients consult when they cough: a comparison of consulting and non-consulting patients. Br J Gen Pract 1998; 48: 1751-1754.

32 Crawford B, Monz B, Hohlfeld J, et al. Development and validation of a cough and sputum assessment questionnaire. Respir Med 2008; 102: 1545-1555.

33 Decalmer SC, Webster D, Kelsall AA, et al. Chronic cough: how do cough reflex sensitivity and subjective assessments correlate with objective cough counts during ambulatory monitoring? Thorax 2007; 62: 329-334.

34 Everett CF, Kastelik JA, Thompson RH, et al. Chronic persistent cough in the community: a questionnaire survey. Cough 2007; 3: 5.

35 Everett CF, Morice AH. Clinical history in gastroesophageal cough. Respir Med 2007; 101: 345-348.

36 Faruqi S, Murdoch RD, Allum F, et al. On the definition of chronic cough and current treatment pathways: an international qualitative study. Cough 2014; 10: 5.

37 Faruqi S, Thompson R, Wright C, et al. Quantifying chronic cough: objective versus subjective measurements. Respirology 2011; 16: 314-320.

38 Ford AC, Forman D, Moayyedi P, et al. Cough in the community: a cross sectional survey and the relationship to gastrointestinal symptoms. Thorax 2006; 61: 975-979.

39 French CL, Crawford SL, Bova C, et al. Change in psychological, physiological, and situational factors in adults after treatment of chronic cough. Chest 2017; 152: 547-562.

40 French CL, Irwin RS, Curley FJ, et al. Impact of chronic cough on quality of life. Arch Intern Med 1998; 158: 1657-1661.

41 Fujimura M. Frequency of persistent cough and trends in seeking medical care and treatment-results of an internet survey. Allergol 2012; 61: 573-581.

42 Hilton E, Marsden P, Thurston A, et al. Clinical features of the urge-to-cough in patients with chronic cough. Respir Med 2015; 109: 701-707. 
43 Hulme K, Dogan S, Parker SM, et al. "Chronic cough, cause unknown": a qualitative study of patient perspectives of chronic refractory cough. J Health Psychol 2019; 24: 707-716.

44 Kang SY, Won HK, Lee SM, et al. Impact of cough and unmet needs in chronic cough: a survey of patients in Korea. Lung 2019; 197: 635-639.

45 Kelsall A, Houghton LA, Jones $\mathrm{H}$, et al. A novel approach to studying the relationship between subjective and objective measures of cough. Chest 2011; 139: 569-575.

46 Kelsall A, Decalmer S, Webster D, et al. How to quantify coughing: correlations with quality of life in chronic cough. Eur Respir J 2008; 32: 175-179.

47 Koskela HO, Latti AM, Pekkanen J. The impacts of cough: a cross-sectional study in a Finnish adult employee population. ERJ Open Res 2018; 4: 00113-2018.

48 Koskela HO, Latti AM, Pekkanen J. Risk factors for repetitive doctor's consultations due to cough: a cross-sectional study in a Finnish employed population. BMJ Open 2019; 9: e030945.

49 Kuzniar TJ, Morgenthaler TI, Afessa B, et al. Chronic cough from the patient's perspective. Mayo Clin Proc 2007; 82: 56-60.

50 Lai $\mathrm{K}$, Zhan W, Li H, et al. The predicative clinical features associated with chronic cough that has a single underlying cause. J Allergy Clin Immunol Pract 2020; 18: 18.

51 Lan NSH, Moore I, Lake F. Understanding cough in interstitial lung disease: a cross-sectional study on the adequacy of treatment. Intern Med J 2021; 51: 923-929.

52 Landt E, Colak Y, Lange P, et al. Chronic cough in individuals with COPD: a population-based cohort study. Chest 2020; 157: 1446-1454.

53 Latti AM, Pekkanen J, Koskela HO. Defining the risk factors for acute, subacute and chronic cough: a cross-sectional study in a Finnish adult employee population. BMJ Open 2018; 8: e022950.

54 Lee KK, Ward K, Rafferty GF, et al. The intensity of voluntary, induced, and spontaneous cough. Chest 2015; 148: 1259-1267.

55 Lee KK, Savani A, Matos S, et al. Four-hour cough frequency monitoring in chronic cough. Chest 2012; 142 : $1237-1243$.

56 Lee KK, Matos S, Ward K, et al. Sound: a non-invasive measure of cough intensity. BMJ Open Respir Res 2017 4: e000178.

57 Ma W, Yu L, Wang Y, et al. Changes in health-related quality of life and clinical implications in Chinese patients with chronic cough. Cough 2009; 5: 7.

58 Mello CJ, Irwin RS, Curley FJ. Predictive values of the character, timing, and complications of chronic cough in diagnosing its cause. Arch Intern Med 1996; 156: 997-1003.

59 Morice AH, Faruqi S, Wright CE, et al. Cough hypersensitivity syndrome: a distinct clinical entity. Lung 2011; 189: 73-79.

60 Murry T, Branski RC, Yu K, et al. Laryngeal sensory deficits in patients with chronic cough and paradoxical vocal fold movement disorder. Laryngoscope 2010; 120: 1576-1581.

61 Ojoo JC, Everett CF, Mulrennan SA, et al. Management of patients with chronic cough using a clinical protocol: a prospective observational study. Cough 2013; 9: 2.

62 Ozge $\mathrm{C}$, Atis S, Ozge A, et al. Cough headache: frequency, characteristics and the relationship with the characteristics of cough. Eur J Pain 2005; 9: 383-388.

63 Sato R, Handa T, Matsumoto $\mathrm{H}$, et al. Clinical significance of self-reported cough intensity and frequency in patients with interstitial lung disease: a cross-sectional study. BMC Pulm Med 2019; 19: 247.

64 Shariat SF, Zimmern PE, Hilton K, et al. Prospective questionnaire-based evaluation of the prevalence of urinary incontinence in women with chronic cough. Urol Int 2009; 83: 181-186.

65 Shembel AC, Rosen CA, Zullo TG, et al. Development and validation of the cough severity index: a severity index for chronic cough related to the upper airway. Laryngoscope 2013; 123: 1931-1936.

66 Sinha A, Porter T, Wilson A. The use of online health forums by patients with chronic cough: qualitative study. J Med Internet Res 2018; 20: e19.

67 Smith J, Owen E, Earis J, et al. Cough in COPD: correlation of objective monitoring with cough challenge and subjective assessments. Chest 2006; 130: 379-385.

68 Song WJ, Morice AH, Kim MH, et al. Cough in the elderly population: relationships with multiple comorbidity. PLoS One 2013; 8: e78081.

69 Ternesten-Hasseus E, Larsson S, Millqvist E. Symptoms induced by environmental irritants and health-related quality of life in patients with chronic cough - a cross-sectional study. Cough 2011; 7: 6 .

70 Van de Kerkhove C, Goeminne PC, Van Bleyenbergh P, et al. A cohort description and analysis of the effect of gabapentin on idiopathic cough. Cough 2012; 8: 9.

71 Vazquez Guillamet R, Petersen H, Meek P, et al. Grading severity of productive cough based on symptoms and airflow obstruction. COPD 2018; 15: 206-213.

72 Vertigan AE, Bone SL, Gibson PG. Development and validation of the Newcastle laryngeal hypersensitivity questionnaire. Cough 2014; 10: 1. 
73 Vertigan AE, Theodoros DG, Winkworth AL, et al. Perceptual voice characteristics in chronic cough and paradoxical vocal fold movement. Folia Phoniatr Logop 2007; 59: 256-267.

74 Vertigan AE, Theodoros DG, Gibson PG, et al. Voice and upper airway symptoms in people with chronic cough and paradoxical vocal fold movement. J Voice 2007; 21: 361-383.

75 Vertigan AE, Gibson PG. Chronic refractory cough as a sensory neuropathy: evidence from a reinterpretation of cough triggers. J Voice 2011; 25: 596-601.

76 Won HK, Kang SY, Kang Y, et al. Cough-related laryngeal sensations and triggers in adults with chronic cough: symptom profile and impact. Allergy Asthma Immunol Res 2019; 11: 622-631.

77 Xu X, Yang Z, Chen Q, et al. Comparison of clinical characteristics of chronic cough due to non-acid and acid gastroesophageal reflux. Clin Respir J 2015; 9: 196-202.

78 Fletcher KE, French CT, Irwin RS, et al. A prospective global measure, the Punum Ladder, provides more valid assessments of quality of life than a retrospective transition measure. $J$ Clin Epidemiol 2010; 63: 1123-1131.

79 Fontana GA, Widdicombe J. What is cough and what should be measured? Pulm Pharmacol Ther 2007; 20: 307-312.

80 Cho PSP, Fletcher HV, Turner RD, et al. Impaired cough suppression in chronic refractory cough. Eur Respir J 2019; 53: 1802203.

81 Hilton E, Satia I, Holt K, et al. The effect of pain conditioning on experimentally evoked cough: evidence of impaired endogenous inhibitory control mechanisms in refractory chronic cough. Eur Respir J 2020; 56: 2001387.

82 Leech J, Mazzone SB, Farrell MJ. Brain activity associated with placebo suppression of the urge-to-cough in humans. Am J Respir Crit Care Med 2013; 188: 1069-1075.

83 Ando A, Smallwood D, McMahon M, et al. Neural correlates of cough hypersensitivity in humans: evidence for central sensitisation and dysfunctional inhibitory control. Thorax 2016; 71: 323-329.

84 Abubakar AB, Bautista TG, Dimmock MR, et al. Behavioral and regional brain responses to inhalation of capsaicin modified by painful conditioning in humans. Chest 2021; 159: 1136-1146. 